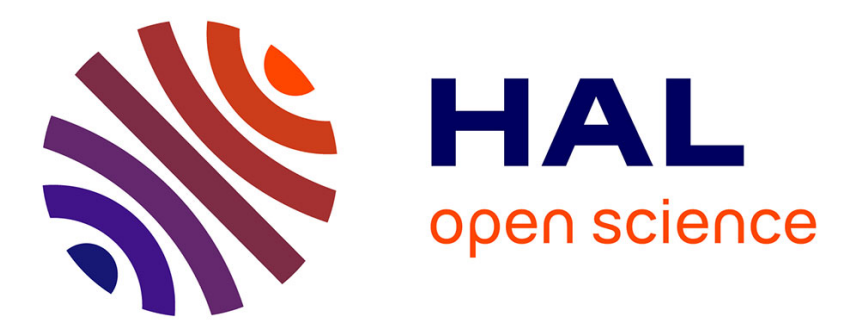

\title{
On the similarity of plastic flow processes during smooth and jerky flow: Statistical analysis
}

M.A. Lebyodkin, N.P. P Kobelev, Y. Bougherira, Denis Entemeyer, C. Fressengeas, V.S. S Gornakov, T.A. A Lebedkina, I.V. V Shashkov

\section{- To cite this version:}

M.A. Lebyodkin, N.P. P Kobelev, Y. Bougherira, Denis Entemeyer, C. Fressengeas, et al.. On the similarity of plastic flow processes during smooth and jerky flow: Statistical analysis. Acta Materialia, 2012, 60 (9), pp.3729-3740. 10.1016/j.actamat.2012.03.026 . hal-02173954

\section{HAL Id: hal-02173954 \\ https://hal.science/hal-02173954}

Submitted on 21 Jul 2019

HAL is a multi-disciplinary open access archive for the deposit and dissemination of scientific research documents, whether they are published or not. The documents may come from teaching and research institutions in France or abroad, or from public or private research centers.
L'archive ouverte pluridisciplinaire $\mathbf{H A L}$, est destinée au dépôt et à la diffusion de documents scientifiques de niveau recherche, publiés ou non, émanant des établissements d'enseignement et de recherche français ou étrangers, des laboratoires publics ou privés. 


\title{
On the similarity of plastic flow processes during smooth and jerky flow: Statistical analysis
}

\author{
M.A. Lebyodkin ${ }^{a}$, N. P. Kobelev ${ }^{b}$, Y. Bougherira ${ }^{a}$, D. Entemeyer ${ }^{a}$, C. Fressengeas ${ }^{a}$, \\ V.S. Gornakov ${ }^{\mathrm{b}}$, T.A. Lebedkina ${ }^{\mathrm{b}}$, I.V. Shashkov ${ }^{\mathrm{a}, \mathrm{b}}$ \\ ${ }^{a}$ LEM3 (Laboratoire d'Etude des Microstructures et de Mécanique des Matériaux), Université Paul Verlaine-Metz, \\ CNRS UMR 7239, Ile du Saulcy, 57045 Metz, France \\ ${ }^{b}$ Institute of Solid State Physics RAS, 142432 Chernogolovka, Russia
}

\begin{abstract}
Jerky flow in dilute alloys, or the Portevin-Le Chatelier effect, is investigated using statistical analysis of time series characterizing the evolution of the plastic activity at distinct scales of observation, namely, the macroscopic scale of stress serrations and a mesoscopic scale pertaining to the accompanying acoustic emission. Whereas the stress serrations display various types of statistical distributions depending on the driving strain rate, including power-law, peaked and bimodal histograms, it is found that acoustic emission is characterized by power-law statistics of event size in all experimental conditions. The latter reflect intermittency and self-organization of plastic activity at a mesoscopic scale. This shift in the observed dynamics when the observation length scale is decreased is discussed in terms of the synchronization of small-scale events.
\end{abstract}

Keywords: Portevin-Le Chatelier effect; Dislocation dynamics; Acoustic methods; Self-organization; Synchronization

\section{Introduction}

Jerky flow, or the Portevin-Le Chatelier (PLC) effect, is a plastic instability of dilute alloys [1]. It offers a striking example of the complex spatiotemporal dynamics that may arise from the collective behavior of defect populations. Extensively studied during the last decades from the point of view of nonlinear dynamical systems (e.g. [2]), its dynamics was shown to be controlled by three basic ingredients, namely: a threshold character, the separation of two (slow vs. fast) time scales, and spatial coupling between the participating defects. All of these features are common to dynamical systems displaying self-organized criticality (SOC) [3] or synchronization [4]. The threshold character derives from the microscopic mechanism of the PLC effect, which is generally believed to be the dynamic strain aging (DSA) of dislocations, i.e. the process by which dislocations arrested at obstacles, and waiting for thermal activation of their motion, are additionally pinned by 
diffusing solute atoms [2,5]. Such extra pinning may lead to negative sensitivity of the flow stress $\sigma$ to the plastic strain rate $\dot{\varepsilon}$ in a certain temperature and $\dot{\varepsilon}$ range, which results in an $N$-shaped dependence $\sigma(\dot{\varepsilon})$ for a given temperature. This relationship plays in plasticity a role similar to the velocity dependence of the friction force in stick-slip processes - a model object for SOC studies [6]. Besides a threshold stress for plastic activity, it implies the existence of two distinct time scales. The fast time scale corresponds to the sudden $\dot{\varepsilon}$ jumps undergone by the system upon reaching the threshold stress. In velocity-driven experiments with a constant imposed overall strain rate $\dot{\varepsilon}_{a}$, the elastic unloading accompanying these jumps manifests itself by sharp drops in the applied force. The slow time scale is the characteristic duration of the subsequent reloading stages. The presence of fast vs. slow time scales is typical of relaxation oscillations [7]. By neglecting plastic strain heterogeneity and spatial coupling, early models of the PLC effect described accordingly the dynamics in terms of relaxation oscillations [8,9]. However, the extensive range of the observed spatiotemporal patterns, which includes localization of strain in narrow bands and propagation of these bands along the sample axis, could not be predicted by these local formulations. Several nonlocal models have been since providing interpretation for the observed spatiotemporal patterns, by taking spatial coupling between system elements into account [10-15].

Various approaches to the experimental analysis of the stress drops were proposed [10,16-21]. They all showed that spatiotemporal patterning corresponds to nontrivial dynamical regimes. In particular, power-law statistical distributions were found for stress serrations in a range of high $\dot{\varepsilon}_{a}$ [10,16-18]. Such scale-free behavior is characteristic of avalanche processes and bears witness to SOC-like dynamics, consistent with the infinite number of degrees of freedom of the dislocation ensembles. Histograms with characteristic peaks were observed for lower strain rates. They were shown to be associated not with stochastic behavior, but with low-dimensional deterministic chaos $[2,17,18]$. Chaos is also characterized by scale invariance, which is reflected in the geometry of the system attractor, as reconstructed from the stress-time series.

Observing scale invariance in the distributions of stress serrations at the macroscopic level, suggests extending the analysis to finer event scales by using more sensitive techniques, e.g. by recording the accompanying acoustic emission (AE) [22-24] or electric signals [25-27]. The statistics of the $\mathrm{AE}$ was studied rather in detail during macroscopically homogeneous deformation of some pure crystalline solids, such as ice and copper [23,28,29]. In contrast to the conventional viewpoint, which contends that smooth plastic flow stems from stochastic motion averaging out in systems containing large numbers of dislocations, the observed AE was not described by Gaussian statistics, but displayed power-law distributions as well. This result bears evidence to the intermittent scale-invariant character of the (macroscopically uniform) plastic activity, albeit at 
sizes of the local strain-rate jerks much smaller than in jerky flow. Power-law statistics were also found for the $\mathrm{AE}$ accompanying twinning of single crystals of $\mathrm{Zn}$ and $\mathrm{Cd}$ [29], for local strain-rate bursts detected by high-resolution extensometry during plastic flow of $\mathrm{Cu}$ single crystals [30], and for stress serrations observed in the compression of microscopic pillars of pure metals [31]. The collection of all the above-described results led to the growing recognition of a ubiquitous character of self-organization phenomena in dislocation ensembles. The various data on small-scale intermittency in pure single crystals provided approximately the same value for the power-law exponent, thus giving rise to a "universality" conjecture [32]. Concurrently, the exponent values reported for AE in polycrystals [33] or for stress drops in the PLC effect [16,34] were found to depend on the experimental conditions and microstructures.

AE was also monitored in solute alloys displaying the PLC effect, namely for Al alloys [22,3537]. However, the statistical analysis of AE data has not been carried out, except for a recent short communication [24]. Such investigation is particularly interesting for several reasons. First, several scales of strain-rate discontinuity are concurrently available. The macroscopic ones are related with the instability phenomenon itself, while the smaller ones also contribute to the regular stable plasticity flow processes. Therefore, supplementing mechanical testing with the AE techniques allows accounting for both scales in a single analysis, whereas the smaller scale usually remains unresolved in traditional mechanical testing. Second, the onset of plastic instability needs a certain critical strain $\varepsilon_{c r}[38,39]$. Comparing $\mathrm{AE}$ records obtained during the macroscopically smooth flow before the onset of instability and during the PLC effect is an opportunity which may allow contrasting (or likening) the strain-rate jerks observed at the different scales. Finally, the abovementioned richness of its dynamics, and the convenience of its practical setup make the PLC effect a model case study of general phenomena encountered in nonlinear systems.

In our previous paper [40], application of the multifractal analysis to the AE accompanying unstable plastic deformation of an AlMg alloy yielded the unexpected result that the temporal correlations characterizing the AE show no peculiarities associated with the PLC effect. The key to understanding such overall similarity of the mesoscopic scale deformation processes during both stress serrations and smooth plastic flow is provided by the comparative statistical analysis of the deformation curves and the accompanying $\mathrm{AE}$, reported in the present work. Its aim is to understand the possible effects of the scale of observation on the observed dynamics. The paper is organized as follows. Section 2 describes the experimental setup. Section 3.1 presents the macroscopic mechanical behavior associated with the stress fluctuations and compares it with earlier data. Section 3.2 establishes correspondence between the macroscopic scale of stress fluctuations and the mesoscopic scale uncovered by acoustic emission and confronts the respective 
statistical behaviors. The outline and the general discussion of the obtained data are given in Section 4. Concluding remarks follow.

\section{Experimental procedure and data processing}

Dog-bone-shaped specimens with a gauge part $25 \times 6.8 \times 2.5 \mathrm{~mm}^{3}$ were cut from a cold-rolled sheet of polycrystalline Al-3\% Mg alloy. All specimens were subjected to solid-solution treatment through a usual procedure of annealing at $400{ }^{\circ} \mathrm{C}$ for $2 \mathrm{~h}$, followed by quenching into water [16,39]. Using optical and scanning electron microscopy, no cracks were discerned either on the side surface of the deformed specimens or in the interior of the ductile dimples observed on the fracture surface, which indicates efficient dissolution of second-phase inclusions. Tensile tests were conducted at room temperature with a constant pulling velocity selected in a large interval corresponding to the initial values of $\dot{\varepsilon}_{a}=2 \times 10^{-5} \mathrm{~s}^{-1}-6 \times 10^{-3} \mathrm{~s}^{-1}$. Three to six specimens were tested for each $\dot{\varepsilon}_{a}$. The sampling time for recording the stress-time curves was chosen in a range from $4 \mathrm{~ms}$ for the fastest tests lasting $\sim 60 \mathrm{~s}$ and to $500 \mathrm{~ms}$ for the slowest ones lasting from $5 \mathrm{~h}$ to $7 \mathrm{~h}$. Consequently, all records contained a similar amount of data, $\sim 15,000-50,000$ data points.

The AE was recorded during tensile tests by a computercontrolled Physical Acoustics LOCAN 320 system. The piezoelectric transducer with a frequency band of $100-600 \mathrm{kHz}$ was clamped to the greased surface just above the deforming part of the sample. In contrast to recent data-streaming techniques allowing for continuous measurement of the acoustic signal during short time intervals of typically tens of seconds (see, e.g. Ref. [41]), the LOCAN 320 equipment is suitable for long tests during which it picks out acoustic events (hits) exceeding a preset threshold voltage and measures their characteristics, such as amplitude, duration, count rate, and energy, using a conventional procedure with several preset time parameters (see, e.g. Ref. [42]). The parameters used and the respective definition of the acoustic event characteristics are schematically illustrated in Fig. 1. The hit definition time was chosen to be similar to that utilized by other authors (e.g. Ref. [36]), in order to have a benchmark for the statistical study performed in the present work. The values of HDT $=$ HLT $=300 \mu$ s were used as a basic set. To evaluate the effect of the possible overlapping of the consecutive $\mathrm{AE}$ events and the data lost on the apparent statistics, control tests were carried out with different settings including the least value of $30 \mu$ s that could be set for HLT and HDT (the same ratio PDT $=0.5 \mathrm{HDT}$ was kept in all tests). It should be noted that this minimum value is larger than the estimate of the maximum return time of the sound waves reflected by the specimen surfaces. Indeed, taking a value of $5 \times 10^{-3} \mathrm{~ms}^{-1}$ for the velocity of sound in $\mathrm{Al}$, the estimate of the travel time of sound along the double specimen length is $\sim 10 \mu$ s. It can thus be 
supposed that the hit echoes effectively decay before the next hit can be captured, and do not cause spurious overlapping. On the other hand, this estimate shows that the recorded event may include echoes, leading to an increase in the apparent value of its duration, but not amplitude.

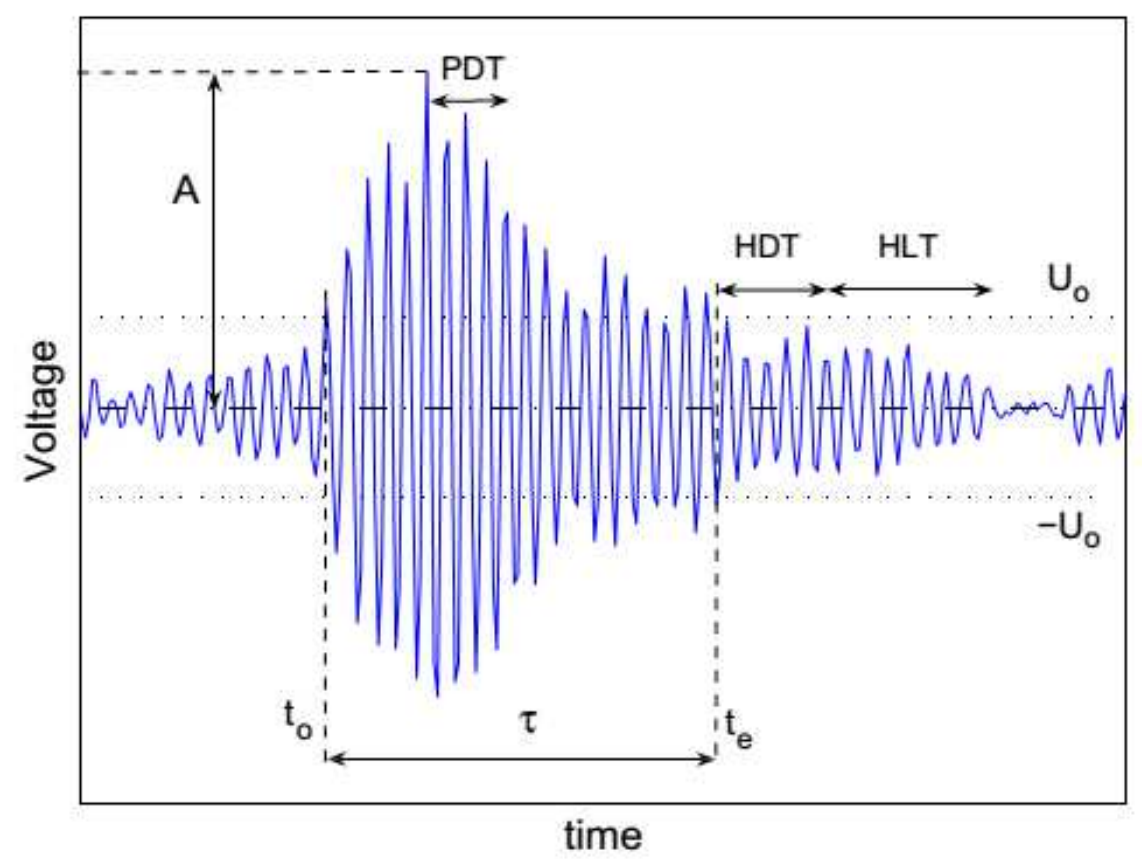

Fig. 1. Definition of the amplitude $A$ and duration $\tau$ for an acoustic event. The acoustic system detects the threshold crossings (counts) and the local maxima of the signal. An event is considered to start at $t=t_{0}$ when the signal exceeds the threshold $U_{0}$. The current maximum is recorded as the event's peak amplitude $A$, provided it was not exceeded during the peak definition time (PDT). Since the amplitude range covers orders of magnitude, the device records the amplitude in the logarithmic scale expressed in decibels, which is designated in the text as $M$. The hit definition time (HDT) allows for the event individualization: if the signal remains below $U_{0}$ during the HDT since the previous threshold crossing $\left(t_{e}\right)$, the event is considered as coming to an end. The hit lockout time (HLT) is a time window during which no measurement is performed after the end of the recorded event. Such a dead time is provided to the system in order to filter out sound reflections. One of its drawbacks is that it may lead to losing useful data.

The total gain of the preamplifier and recording equipment was chosen to be $80 \mathrm{~dB}$ or, in control tests, $95 \mathrm{~dB}$. The threshold voltage for the identification of the starting point of an AE event was set at $27 \mathrm{db}$, which corresponds to the level of the acoustic signal measured in the free-running deforming machine.

As will be described in the corresponding sections, statistical distributions and correlation functions were studied for various measured quantities. For a given quantity $s$, its probability density function is calculated as $P(s)=\frac{1}{\delta s} \frac{\delta N(s)}{N}$, where $N$ is the total number of data in the statistical sample and $\delta N(s)$ is the corresponding histogram with $s$ taken in the middle of the binds. Variable bin sizes are used to handle the statistics of rare events: as a rule of thumb, when an initialsize bin contains less than seven events, it is merged with the next bins until this minimum number is reached (cf. Refs. [10,16]). 
The cross-correlation functions $\rho(\Delta t)$ for two time series, $x_{i}$ and $y_{i}$, representing two quantities measured at the instants $i \delta t(i=1 \ldots n)$, are calculated for positive $\Delta t$ as follows:

$$
\rho(\Delta t)=\frac{\sum_{i=1}^{n-m}\left(x_{i}-\langle x\rangle_{m}\right)\left(y_{i+m}-\langle y\rangle_{m}\right)}{\left(\sum_{i=1}^{n-m}\left(x_{i}-\langle x\rangle_{m}\right)^{2} \cdot \sum_{i=1}^{n-m}\left(y_{i+m}-\langle y\rangle_{m}\right)^{2}\right)^{1 / 2}}
$$

Where $\Delta t=m \delta t, 0 \leq m\left\langle n\right.$, the averages $\langle x\rangle_{m}$ and $\langle y\rangle_{m}$ are taken over the members of the series participating in the sum, and $m$ is taken small with regard to $n$ in order to obtain accurate averages. The time series $x_{i}$ and $y_{i}$ are swapped in this equation to calculate $\rho(\Delta t)$ for negative $\Delta t$.

\section{Experimental results and data analysis}

\subsection{Types and statistics of stress serrations}

First, we describe the well-known attributes of the PLC effect, which are retrieved in the investigated alloy. A salient feature is the gradual transition from the so-called type A to type B to type $\mathrm{C}$ behavior, which takes place when $\dot{\varepsilon}_{a}$ is decreased from the upper to the lower boundary of the range of instability [2]. Examples of stress-strain curves displaying these specific types are given in Fig. 2; mixed behavior was observed at intermediate strain rates. Type A behavior is characterized by stress fluctuations at all scales, which are associated with deformation bands usually nucleating near one specimen end and propagating quasi-continuously along the tensile axis $^{1}$. In type B behavior, rather regular stress drops are reminiscent of relaxation oscillations. Each stress drop results from a static deformation band nucleated ahead of the previous band, for which reason this regime is often referred to as "hopping propagation". As the localized plastic deformation results in material work hardening within the band, the serrations often form groups corresponding to hopping propagation along the entire specimen. Hopping propagation periods are separated by stretches of more regular deformation during which the stress increases, until it finally triggers a new band. Type $\mathrm{C}$ serrations are usually attributed to randomly nucleated bands, although the analysis of the serrations bears witness to the existence of some correlation [44].

In addition to this conventional pattern, type $\mathrm{C}$ and type $\mathrm{B}$ deformation curves often display smaller-scale fluctuations which have received little attention so far. Blowing up type C deformation curves allows distinguishing two separate scales of stress serrations, as shown in Fig. 3. The deep stress drops with amplitude $\Delta \sigma$ in the range 5-15 MPa, occurring beyond the critical strain, are considered a signature of type $\mathrm{C}$ behavior. In addition, small stress drops are occurring almost from the onset of plastic deformation. They increase progressively to 1-2 MPa, a level remaining roughly the same till the end of the test. These small-scale events are strongly dispersed

\footnotetext{
${ }^{1}$ The latter was checked with the aid of the local extensometry technique [43].
} 
above $\varepsilon_{c r}$, and sweep the whole range of intensity down to the noise level of the measurements ( $\sim 0.01 \mathrm{MPa})$. In type B conditions, the initial portion of the deformation curve also displays lowamplitude events, albeit in the form of smooth undulations. These events cannot be clearly isolated from type $\mathrm{B}$ serrations above $\varepsilon_{c r}$ : in contrast to Fig. 3, the amplitudes of all serrations form a continuous cloud of data points. When $\dot{\varepsilon}_{a}$ is further increased (type A behavior), no characteristic scale of serrations is observed.

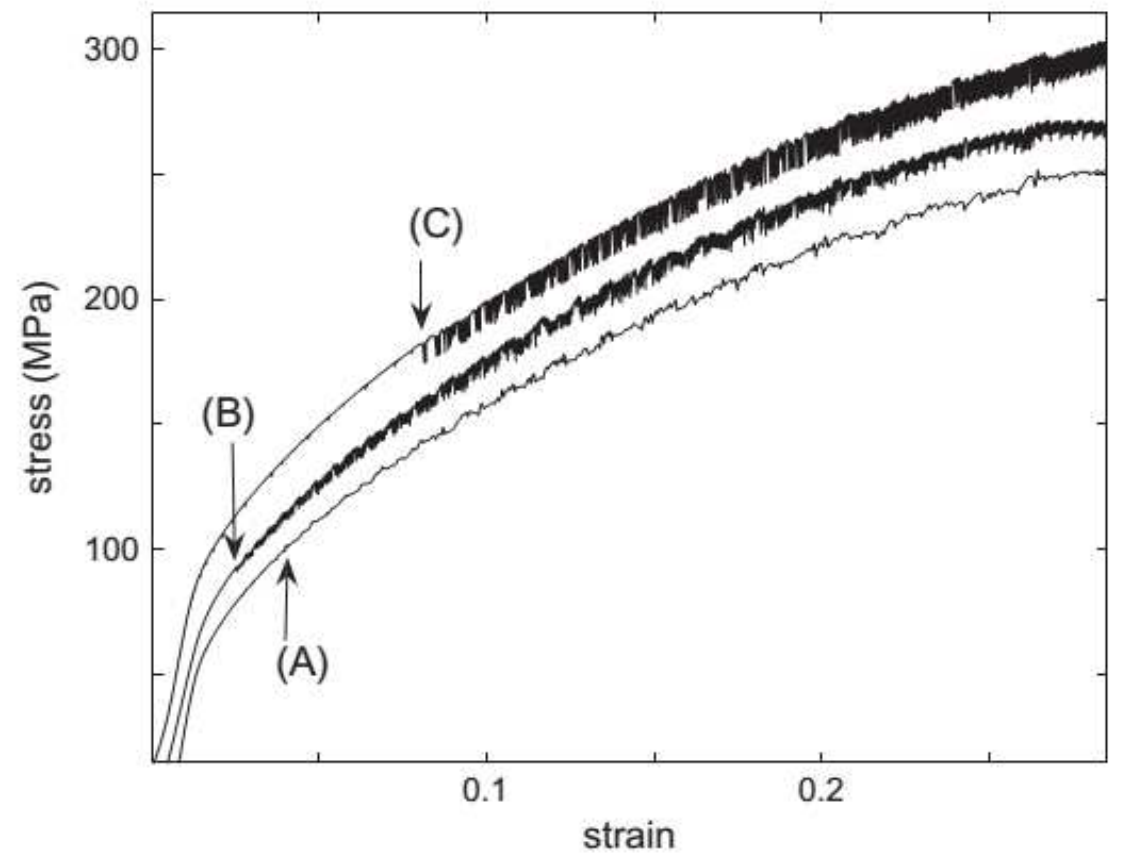

Fig. 2. Examples of true stress-true strain curves for various strain rates at room temperature: (A) $6 \times 10^{-3} \mathrm{~s}^{-1}$, (B) $2 \times 10^{-4} \mathrm{~s}^{-1}$, (C) $2 \times 10^{-5} \mathrm{~s}^{-1}$. The letters in brackets are chosen to designate the corresponding band types A, B and C. The respective arrows indicate the critical strain $\varepsilon_{c r}$ for the onset of plastic instability.

Like the characteristic shapes of the three conventional types of stress serrations, their statistical distributions also display well-known features [2,16,18,39,45]. It should be noticed that work hardening may influence the average magnitude of the serrations, which in such cases evolves during some transient period, to finally stabilize in a close-to-steady state. To calculate the $\Delta \sigma$ distribution, these steady-state intervals are investigated. Besides, in order to reduce the workhardening effect as much as possible, the data are normalized with respect to a low-order polynomial fit. Various normalization procedures, all providing close quantitative results, are described in Refs. $[10,16,18,46]$. In the case when $\Delta \sigma$ fluctuates around a constant average $\langle\Delta \sigma\rangle$ value, the normalization procedure reduces to the ratio $\Delta \sigma /\langle\Delta \sigma\rangle$. Normalization is, however, useful when the statistics are to be compared for different datasets. Consequently, it is systematically applied to the various data in the present work. Scale-free power-law statistics are found for type A serrations, as illustrated in Fig. 4. The type B instability is characterized by large peaked 
M.A. Lebyodkin, N.P. Kobelev, Y. Bougherira, D. Entemeyer, C. Fressengeas, et al.. On the similarity of plastic flow processes during smooth and jerky flow: Statistical analysis. Acta Materialia, Elsevier, 2012, 60, pp.3729-3740. 10.1016/j.actamat.2012.03.026

distributions reflecting the occurrence of a characteristic scale (Fig. 5). The shape of the peaks is usually asymmetrical due to the enhanced probability of low amplitude events. Such a bimodal character of the histograms becomes conspicuous for type $\mathrm{C}$ behavior, so that the distributions clearly disintegrate into two parts [39].
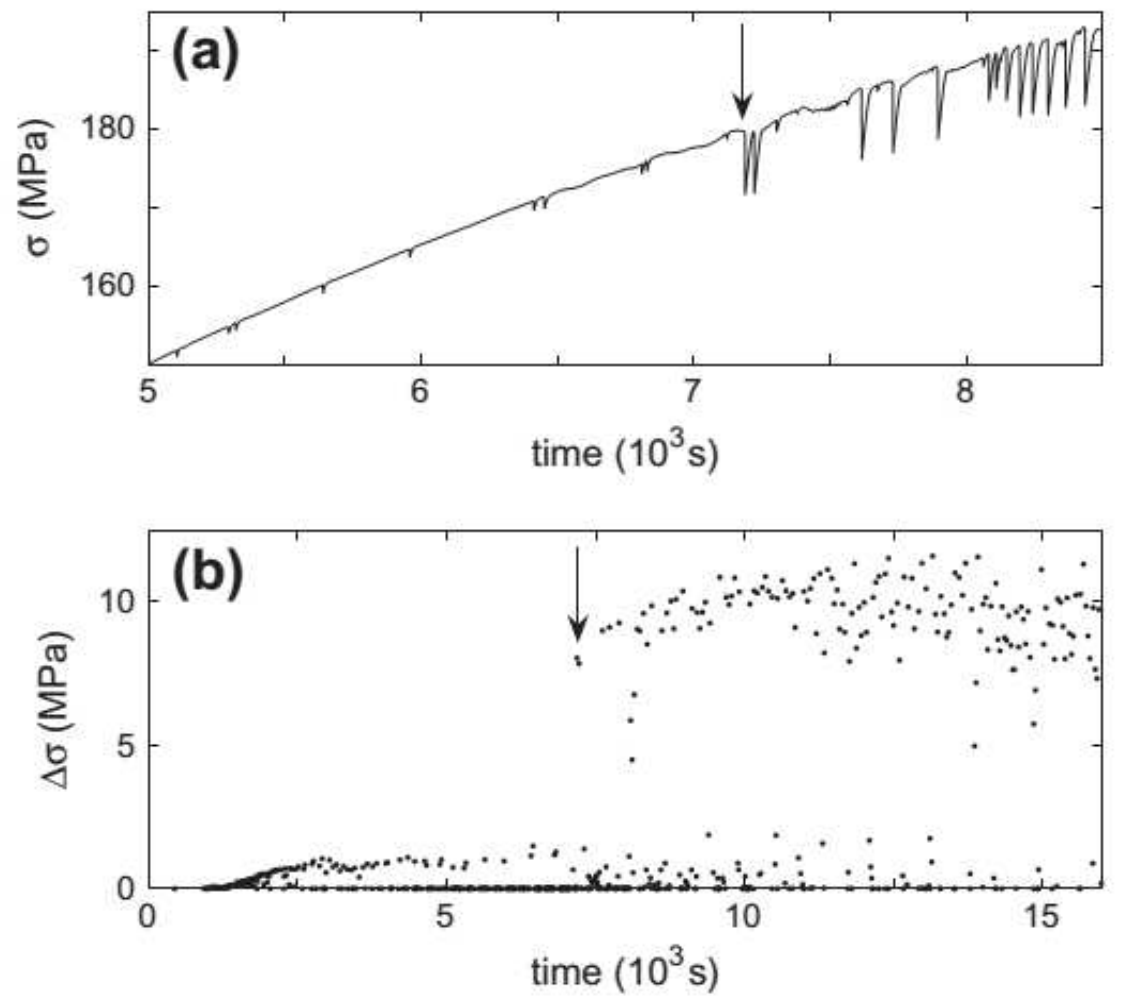

Fig. 3. (a) Magnification of a type $C$ nominal stress vs. time curve. (b) Sequence of amplitudes $\Delta \sigma$ of stress drops for the above deformation curve. The arrows indicate the onset of sharp type $\mathrm{C}$ serrations. $\dot{\varepsilon}_{a}=2 \times 10^{-5} \mathrm{~s}^{-1}$.

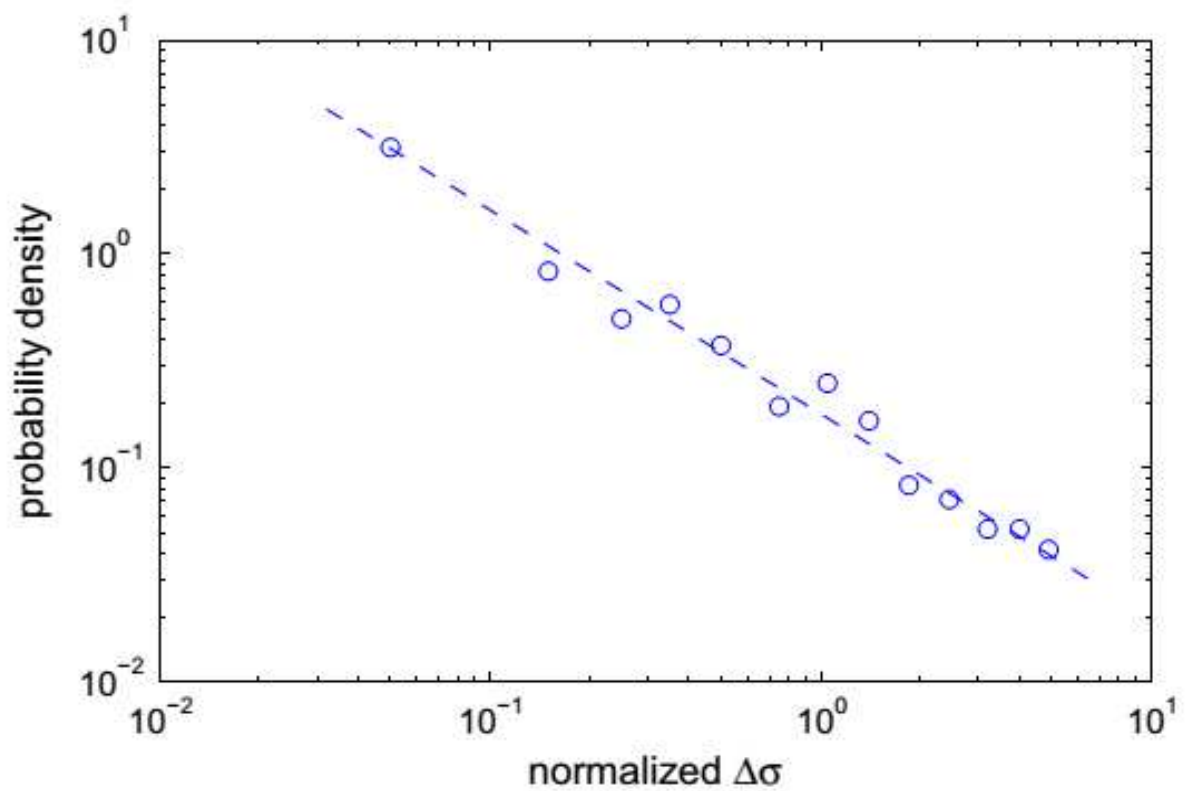

Fig. 4. Probability density function for the amplitude $\Delta \sigma$ of stress drops for a sample deformed at $\dot{\varepsilon}_{a}=2 \times 10^{-3} \mathrm{~s}^{-1}$ (type A behavior). 
M.A. Lebyodkin, N.P. Kobelev, Y. Bougherira, D. Entemeyer, C. Fressengeas, et al.. On the similarity of plastic flow processes during smooth and jerky flow: Statistical analysis. Acta Materialia, Elsevier, 2012, 60, pp.3729-3740. 10.1016/j.actamat.2012.03.026

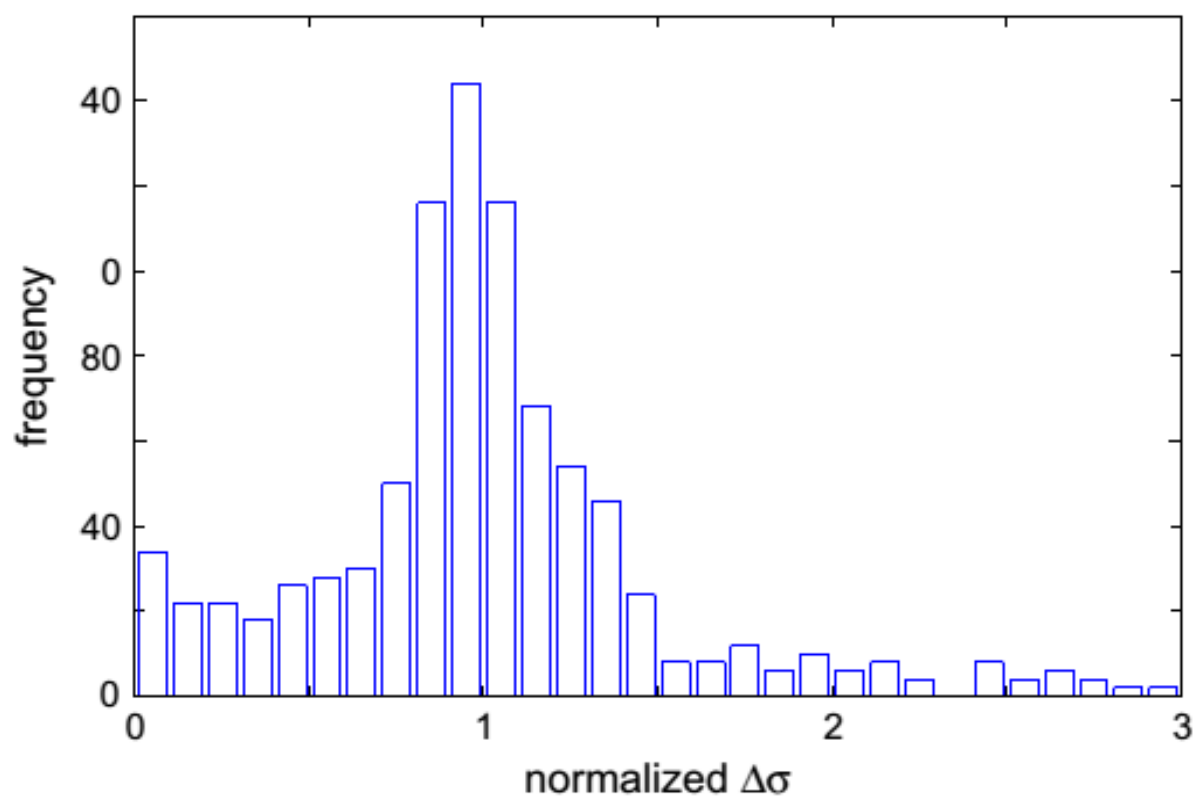

Fig. 5. Histogram of distribution of stress drops for a sample deformed in type B conditions, $\dot{\varepsilon}_{a}=2 \times 10^{-4} \mathrm{~s}^{-1}$.
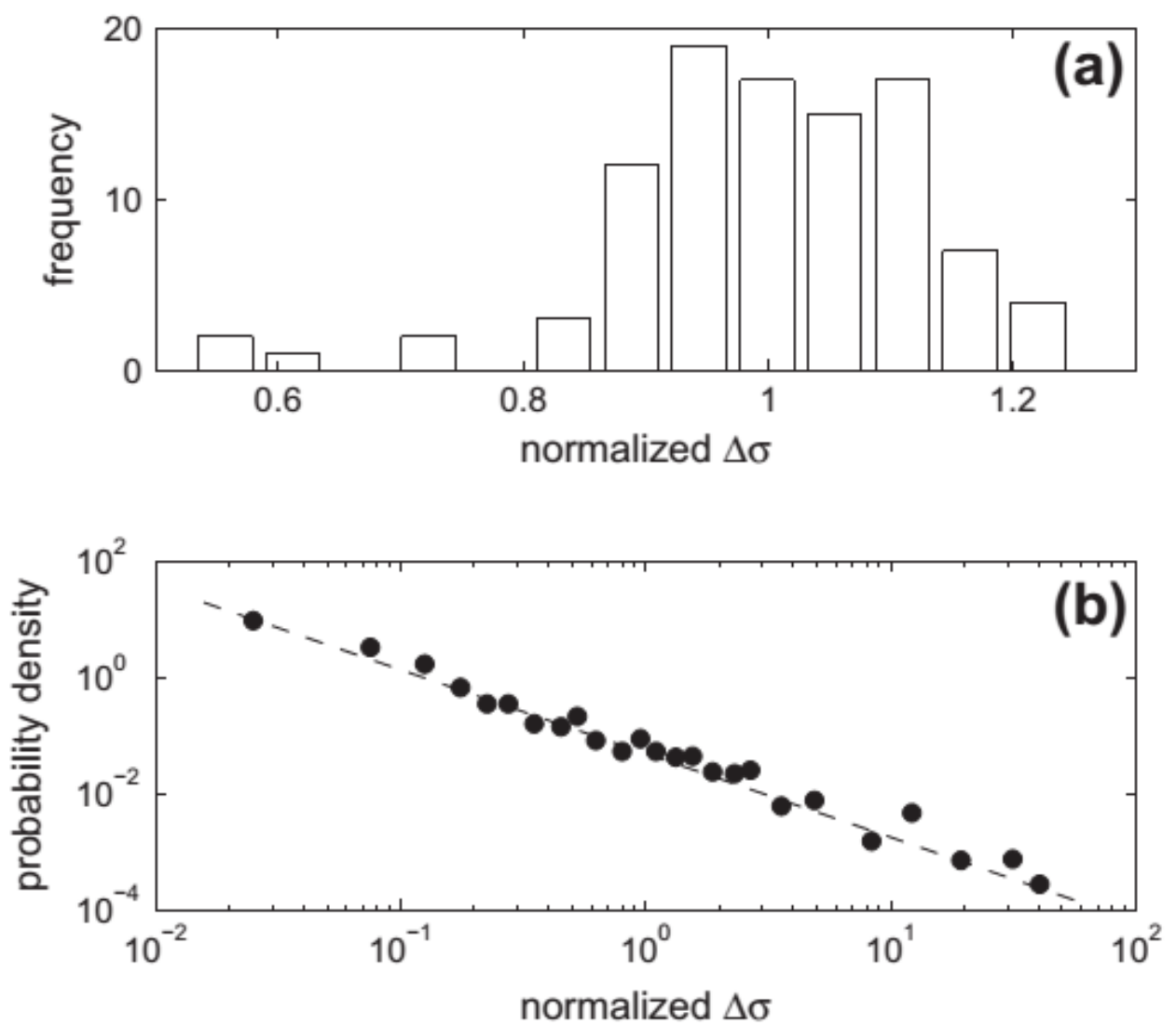

Fig. 6. (a) Histogram of distribution of stress drops of type C, $\dot{\varepsilon}_{a}=2 \times 10^{-5} \mathrm{~s}^{-1}$. (b) Probability density function for low-amplitude stress drops, $\Delta \sigma<5 \mathrm{MPa}$, observed for the same specimen in the same time interval.

An unexpected result is obtained when the statistical analysis is applied separately to these two groups of events (Fig. 6). Whereas rather symmetrical peaked distributions are obtained for the 
large stress drops, in agreement with previous studies, power-law behavior is found for the small serrations observed in the same time interval. It can be seen in Fig. 6b that the dependence $P(\Delta \sigma) \sim \Delta \sigma^{-\alpha}$ is valid in a wide range covering 2-3 orders of magnitude of $\Delta \sigma$. The exponent $\alpha$ found for various specimens varies in the range of 1-1.5, similar to those obtained for type A stress drops. Power-law dependences are also verified for the duration probability distribution, although in a narrower range covering 1-1.5 orders of its magnitude, and for the relationship between the amplitude and duration [47], following from their power-law statistics. At variance with the usual assumption that such small serrations are random in nature $[48,49]$, these observations testify to a critical character of their statistics, even though not necessarily of SOC type. This transition to critical-type statistics when reducing the scale of observation becomes obvious in the analysis of the $\mathrm{AE}$, as will be shown below.

\subsection{Acoustic emission}

\subsubsection{Correlation with the stress instabilities}

The previous studies of the $\mathrm{AE}$ in dynamically strain aging alloys mostly reported on the behavior of the averaged characteristics, such as the count rate which gives the average frequency of acoustic oscillations, i.e. the average number of threshold crossings per second by the acoustic signal $[22,35,36]$. A similar analysis of the present data shows that like the deformation curves, the overall AE behavior is consistent with the literature data. Although the exact dependences may noticeably vary even when the samples are tested in the same conditions, the major characteristics remain the same in all tests and for all $\dot{\varepsilon}_{a}$ values. Namely, AE starts from onset of plastic flow and its activity quickly reaches a maximum, as illustrated in Fig. 7a and b for one of the specimens deformed at $\dot{\varepsilon}_{a}=2 \times 10^{-5} \mathrm{~s}^{-1}$. The initial high AE activity is also typical of pure materials and is usually attributed to intensive dislocation multiplication and long free path of dislocations in the unhardened material. The count rate further decreases and usually reaches an approximately stationary level. The onset of PLC serrations beyond the critical strain $\varepsilon_{c r}$ does not visually affect this level but gives rise to bursts, as seen in Fig. 7b. The latter are rather strongly correlated with type $\mathrm{C}$ serrations but the correlation diminishes when $\dot{\varepsilon}_{a}$ is increased: bursts accompany only a part of type B serrations (cf. Ref. [22]); rare bursts are observed in type A conditions, without overall relationship with stress fluctuations. A theoretical framework explaining such behavior was proposed recently [50].

Allied with the simple premise that the occurrence of a stress drop requires cooperative displacement of hundreds thousands of dislocations, the correlation between the AE count-rate bursts and stress serrations, observed at low enough $\dot{\varepsilon}_{a}$, often leads to the simplistic and recently 
questioned [40,51] vision, that the distinct PLC bands are responsible for intense "discontinuous" $\mathrm{AE}$ whereas the reloading periods following the stress drops are accompanied with a weaker "continuous" AE. In the present work, more detailed information was searched by monitoring the as-recorded AE data, with focus on the amplitude and duration of the AE hits. Unexpectedly, even in the case of type $\mathrm{C}$ serrations, which are characterized by the most conspicuous correlation with the count-rate bursts, onset of the PLC serrations does not affect the amplitude range of AE hits. This is illustrated in Fig. 7c, which represents their logarithmic amplitude $M$. In contrast, bursts in the AE event duration $\tau$ are clearly observed in Fig. $7 \mathrm{~d}$. As seen in the example of this figure, the maximum $\tau$ values reach $5-10 \mathrm{~ms}$ for $\dot{\varepsilon}_{a}=2 \times 10^{-5} \mathrm{~s}^{-1}$ and basic value of HDT $=300 \mu \mathrm{s}$, whereas the background durations vary from $1 \mu \mathrm{s}$ to $1 \mathrm{~ms}$. This observation suggests that the occurrence of the $\mathrm{AE}$ events with very long duration results from the merger of successive hits whose amplitudes do not surpass the usual range.
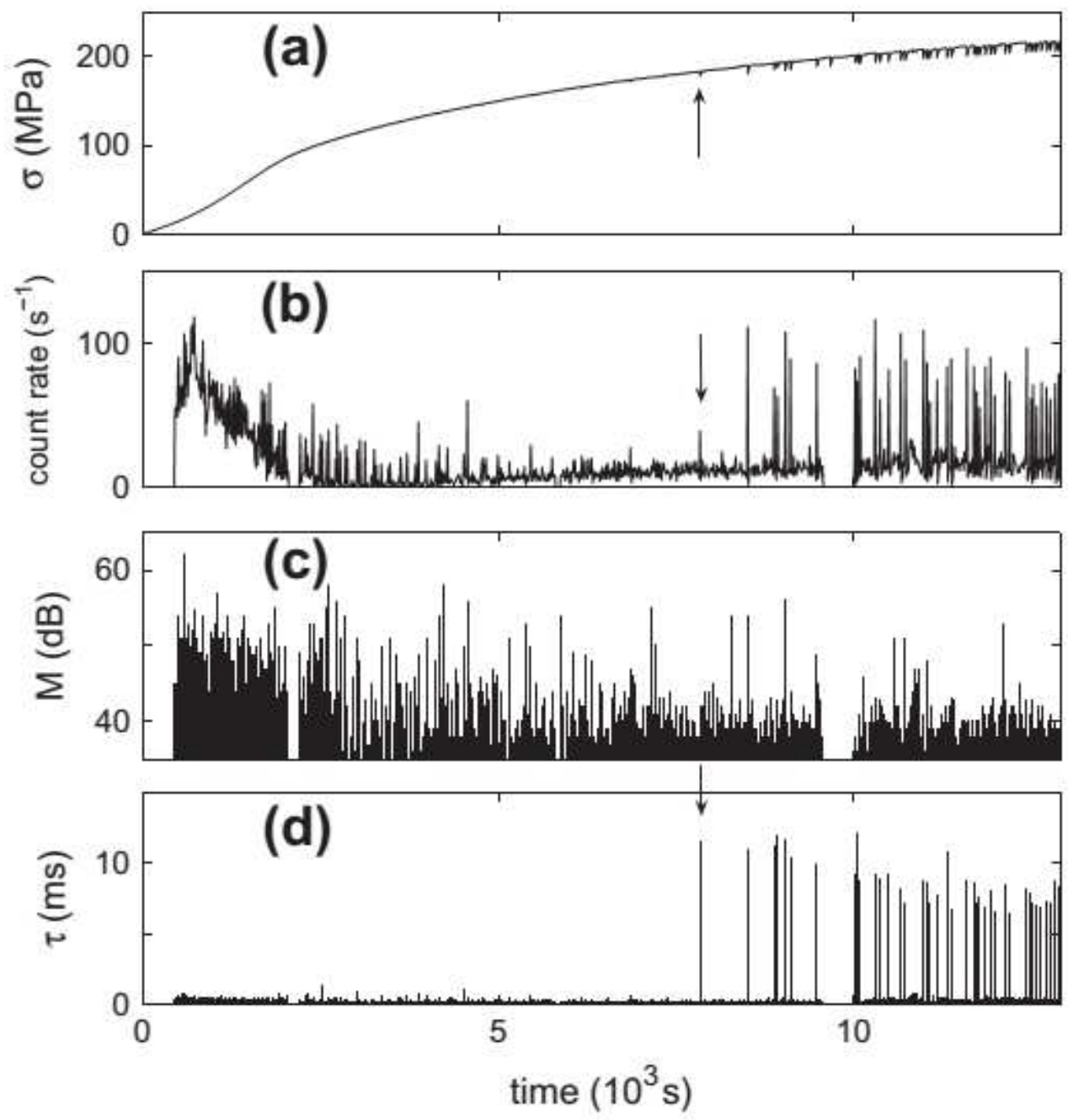

Fig. 7. (a) Nominal stress vs. time curve for the sample of Fig. 3; (b) AE count rate calculated by averaging over time intervals of $10 \mathrm{~s}$; two "silent" intervals correspond to technical stops in AE acquisition; (c) logarithmic amplitude $M$ of the individual AE events; (d) their respective duration $\tau$. HDT $=$ HLT $=300 \mu \mathrm{s}$. The arrows show the instant corresponding to $\varepsilon_{c r}$. 
This conjecture is corroborated by several experimental observations. First, the reduction in HDT does not affect the $M$ range but results in a decrease in $\tau$, which bears evidence to a better individualization of the successive events. For example, when HDT is reduced to $30 \mu$ s in the test at $\dot{\varepsilon}_{a}=2 \times 10^{-5} \mathrm{~s}^{-1}$, the maximum value of $\tau$ bursts decreases to less than $3 \mathrm{~ms}$ and the maximum background level typically becomes as low as $0.2 \mathrm{~ms}$. It should be noticed that the visual pattern remains qualitatively the same and $\tau$ bursts are still present, albeit with a lesser height, which indicates that the merging of events takes place for all device settings. Second, the maximums value becomes higher when $\dot{\varepsilon}_{a}$ is increased. For comparison, the $\tau$ bursts typically reach 10-20 ms (cf. Fig. 11 below) for $\dot{\varepsilon}_{a}=2 \times 10^{-4} \mathrm{~s}^{-1}$ and HDT $=300 \mu \mathrm{s}$. The effect of HDT variation also becomes more significant. Finally, the $\tau$ values practically do not attain a stationary range during the fastest tests $\left(\dot{\varepsilon}_{a}=6 \times 10^{-3} \mathrm{~s}^{-1}\right)$, especially with HDT $=300 \mu \mathrm{s}$. In this latter case the maximum $\tau$ reaches about $100 \mathrm{~ms}$ for the first serrations, and eventually decreases down to a few milliseconds at the end of the test.
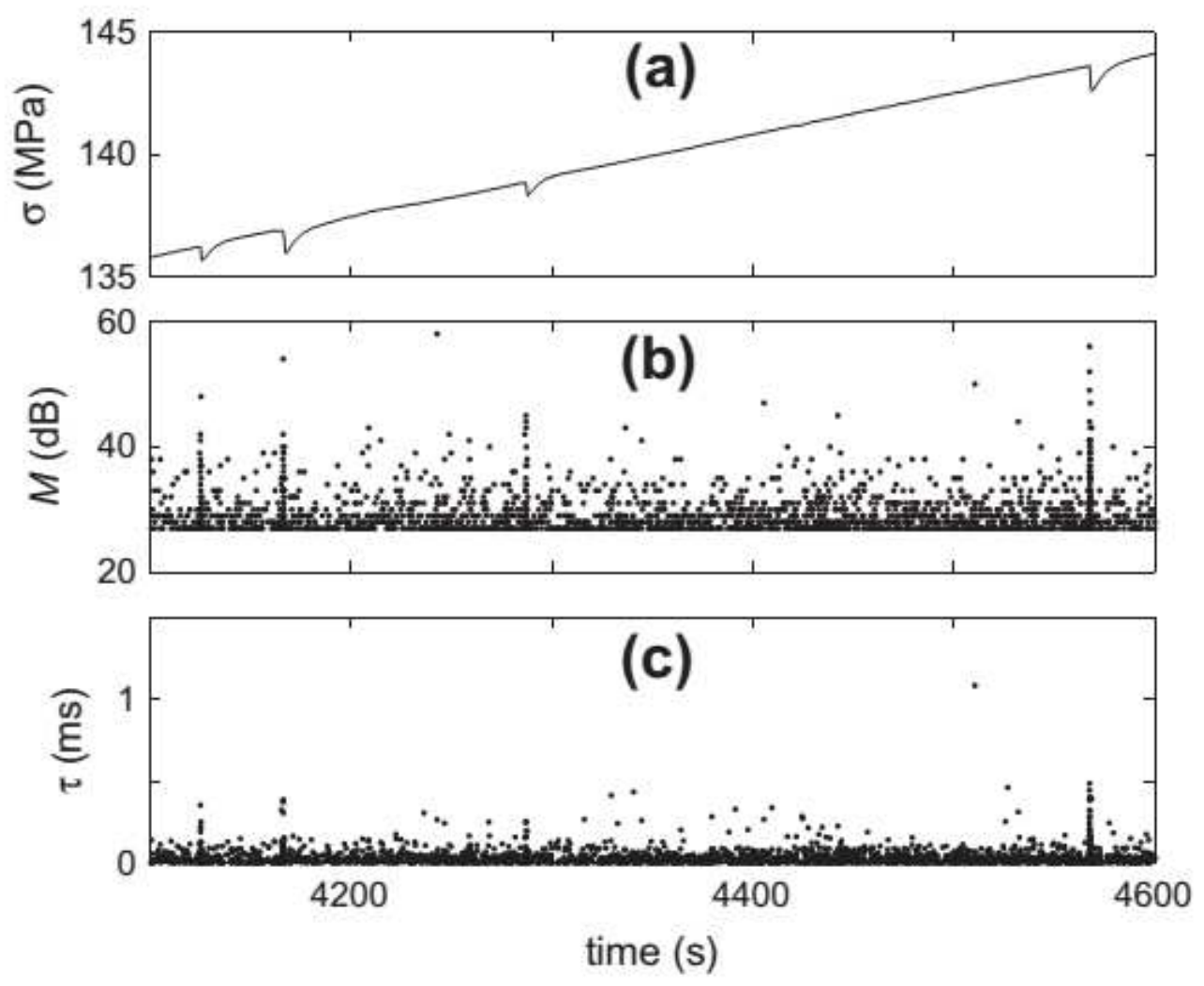

Fig. 8. (a) Blow up of part of a $\sigma(t)$ curve before the onset of type $\mathrm{C}$ serrations. The driving strain rate is $\dot{\varepsilon}_{a}=2 \times$ $10^{-5} \mathrm{~s}^{-1}$. (b) Logarithmic amplitude $M$ of the accompanying AE events. (c) Duration $\tau$ of the accompanying AE events.

Furthermore, the transition from isolated to merged AE hits manifests itself in the details of the correspondence between the stress changes and the $\mathrm{AE}$ at different stages of the deformation 
process. Fig. 8 represents the values of $M$ and $\tau$ for the AE events recorded during the early plastic region, displaying low-amplitude stress drops, of the deformation curve shown in Fig. 7. It can be seen that the AE events with high $M$ and/or $\tau$ are not an exclusive feature of these serrations: such events also occur during macroscopically smooth plastic flow. What is different between these two cases is that the AE shows a tendency to clustering the hits at the instants of stress drops. This activity also gives rise to visible count-rate bursts (cf. Fig. 7b). Further deformation results in a more uniform scatter in $M$, so that the direct correlation between the stress drops and the maximum $M$ values decays closer to $\varepsilon_{c r}$. This behavior does not change beyond $\varepsilon_{c r}$, as illustrated in Fig. 9. Indeed, the $\mathrm{AE}$ events recorded at the instants of the deep type $\mathrm{C}$ serrations and those accompanying the smooth reloading parts correspond to the same $M$ range (except for one specimen occasionally displaying $M$ bursts for several stress drops). On the contrary, the clusters of AE events accompanying stress serrations degenerate into single events with very long duration. More precisely, representation of $\tau$ in the logarithmic scale reveals several more hits with relatively enhanced duration during a stress drop. It should be noted that when HDT is decreased, groups of hits, and not only isolated hits, may again be observed during some of the stress drops.
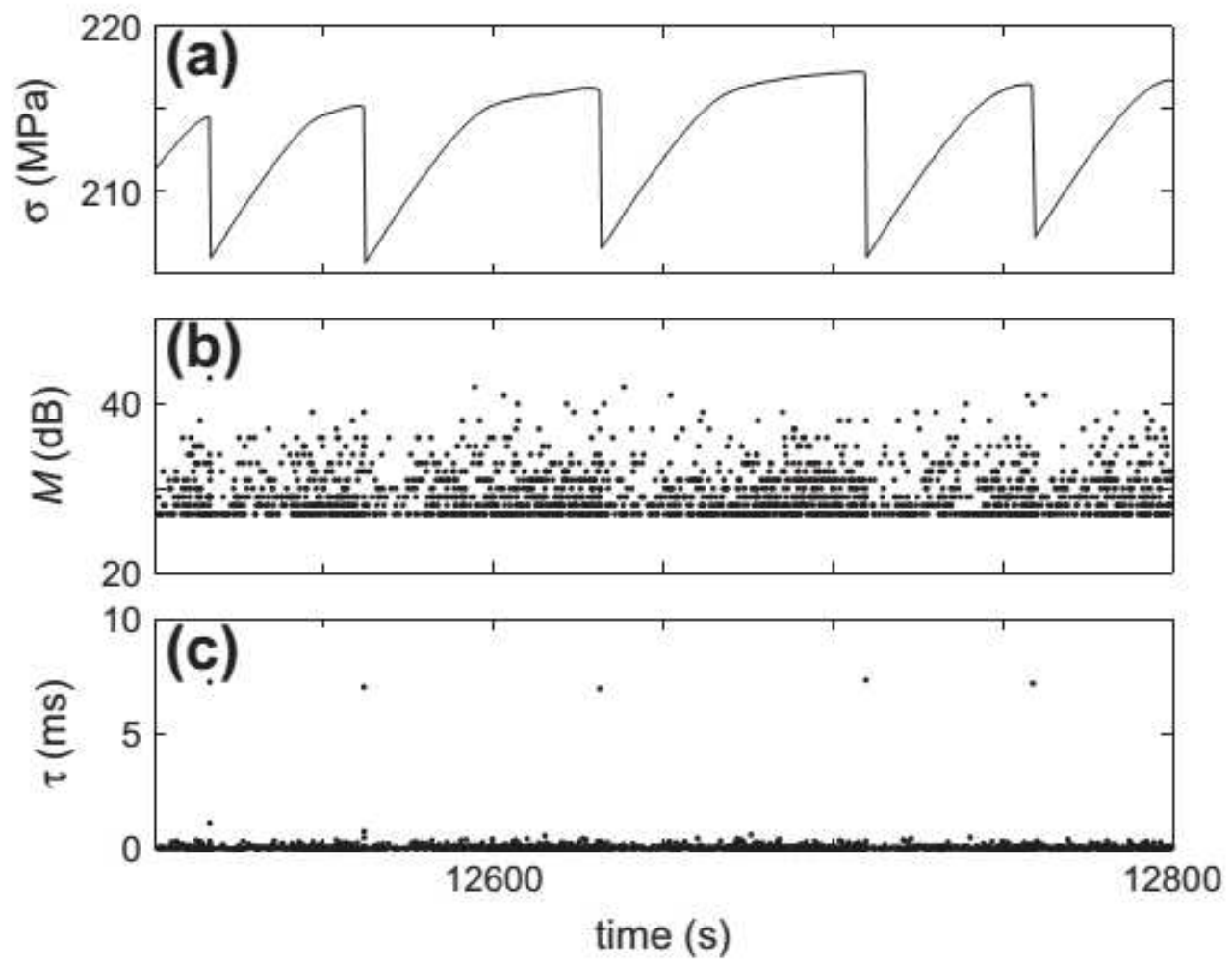

Fig. 9. Same as Fig. 8 for another time interval containing type $\mathrm{C}$ serrations..

These observations imply the same mechanism of plasticity, associated with similar-size dislocation avalanches, at work during both macroscopically stable and unstable flow. In other words, the macroscopic stress serrations are not due to simultaneous motion of unusually large 
dislocation ensembles but to synchronization of ordinary glide events, as suggested in Ref. [40]. This hypothesis also drives the attention to another aspect of the arrangement in time of the AE events, which can be discerned in Fig. 9. Namely, the stress drops are announced by more frequent occurrence of AE events (denser clouds of data points) and are followed by more "silent" periods. Calculation of cross correlations between the deformation curves and the AE confirms this qualitative observation and makes it more precise, displaying rather complex behavior illustrated in Fig. 10. Since the AE is not recorded continuously, the AE time series for the calculation of the correlation are obtained by collecting the AE events in intervals equal to the sampling time $\delta t$ of the deformation curve. So, the cumulated duration $\tau_{\text {cum }}$ results from stacking durations of the $\mathrm{AE}$ events over $\delta t$ and therefore reflects the evolution of the averaged AE activity. In turn, the number of events in $\delta t$ intervals characterizes their clustering in time. Fig. 10 compares the crosscorrelation functions of these time series with the absolute value of the finite-difference approximant of the time derivative of stress, $|\dot{\sigma}(t)|$. The correlation of the latter with $\tau_{\text {cum }}$ is dominated by recurrent groups of peaks reflecting the sequence of stress drops, in conformity with the strong visual correlation between $\tau$ bursts and type $\mathrm{C}$ stress drops. Its correlation with the cumulated number of events also displays oscillations with the same period corresponding to the average frequency of type $\mathrm{C}$ serrations, thus confirming the presence of enriched and impoverished AE before and after stress drops, respectively. This correlation suggests that the plastic instability occurs when the internal stresses reach a high overall level, in consistence with the conjectured mechanism of dislocation avalanche-chaining during a stress drop. Instead, localized overstresses would trigger isolated dislocation avalanches, release isolated $\mathrm{AE}$ hits, and fail to produce macroscopic stress serrations. The correlation manifests more complexity, however, as it also displays shorter-period oscillations. In Fig. 10b, in particular, positive $\rho$ values are also observed between the main maxima associated with the stress serrations, thus indicating clustering of AE hits during the reloading periods.

When the driving strain rate is increased, the direct correlation between the plastic instabilities and the $\tau$ bursts degrades progressively. For comparison, there is no visual correlation between the $\mathrm{AE}$ and the stress undulations below $\varepsilon_{c r}$ for $\dot{\varepsilon}_{a}=2 \times 10^{-4} \mathrm{~s}^{-1}$. Further, the high $\tau$ bursts accompany only part of the type B serrations, as can be seen in Fig. 11, although the presentation of these data in the logarithmic scale reveals some increase in $\tau$ for all serrations. It is worth noting an additional feature often observed for type B instability: more AE hits, some of which have rather high amplitude, are recorded during nucleation of a new sequence of PLC bands than during the adjacent time intervals corresponding to hopping band propagation, as indicated by vertical dotted rectangles in Fig. 11 (cf. Ref. [36]). Since the beginning of a new hopping sequence in the material 
hardened by the previous passage of deformation bands requires a higher overall stress, this observation is consistent with the above conjecture on the clustering of $\mathrm{AE}$ events when the global level of internal stresses increases. Finally, very few AE bursts are observed for type A instability at $6 \times 10^{-3} \mathrm{~s}^{-1}$, and the latter are not clearly correlated with stress fluctuations.
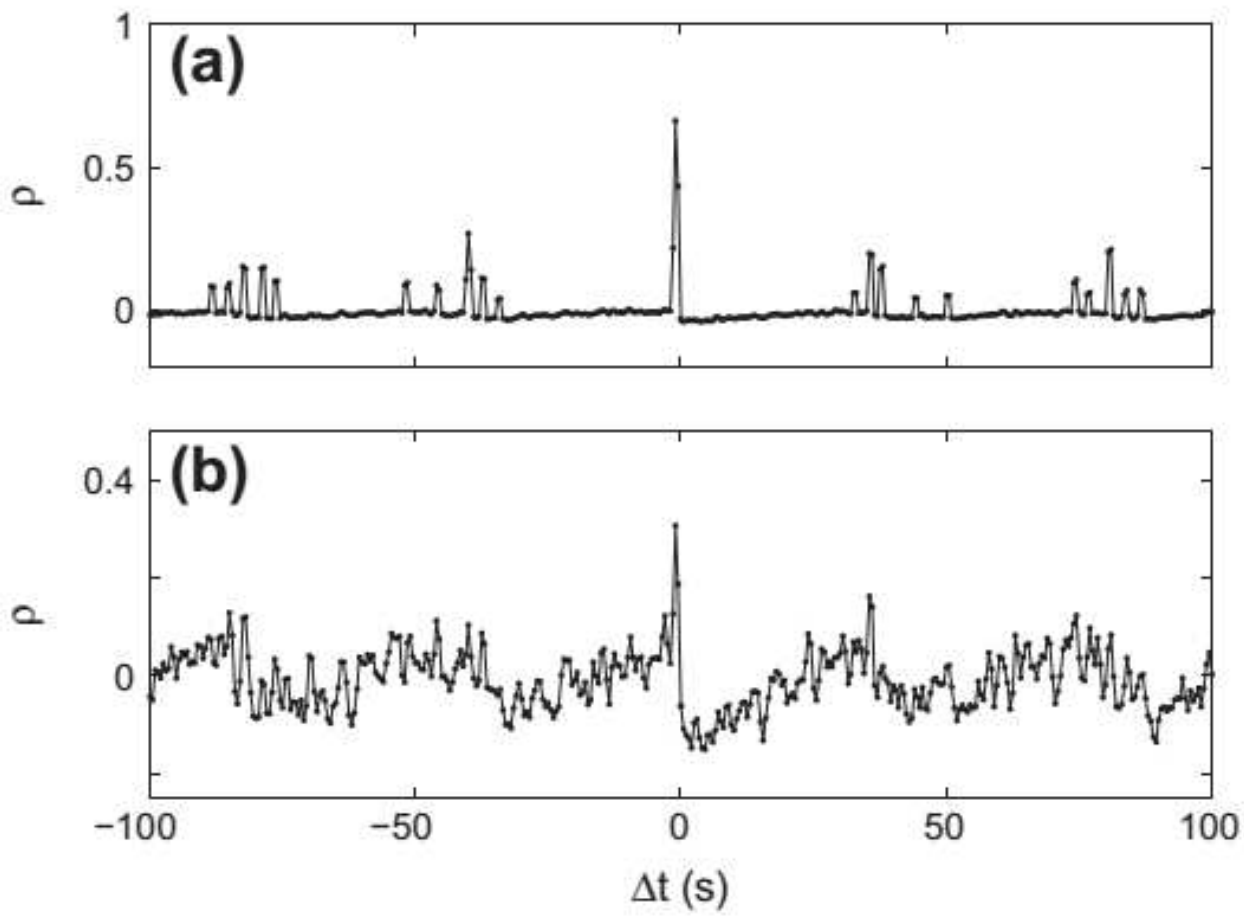

Fig. 10. Cross-correlation functions $\rho(\Delta t)$ for the stress derivative $|\dot{\sigma}(t)|$ and: (a) the cumulative duration $\tau_{\text {cum }}(t)$ of the AE events; (b) the events frequency. The value $\tau_{c u m}$ is obtained by stacking durations over time intervals of $0.5 \mathrm{~s}$ corresponding to the stress sampling time $\delta t$. The events frequency is given by the number of events within $\delta t$ intervals. The driving strain rate is $\dot{\varepsilon}_{a}=2 \times 10^{-5} \mathrm{~s}^{-1}$. Calculation for a time interval featuring type $\mathrm{C}$ serrations $(11,700 \mathrm{~s}, 12,050 \mathrm{~s})$.

\subsubsection{AE statistics}

The statistical analysis of $\mathrm{AE}$ accompanying various physical processes is often restricted to the event amplitude (e.g. Ref. [42]), because the analysis of time characteristics does not properly reflect the properties of the source signal. It is indeed affected by the transfer function of the receiving transducer, the unfiltered sound reflections, the modulations by the propagating medium, etc. Another reason for such a restriction stems from the above results, which indicate that merging of events strongly influences their apparent duration, whereas it is insignificant for the amplitude range. Indeed, even at $\dot{\varepsilon}_{a}=6 \times 10^{-3} \mathrm{~s}^{-1}$, the $\mathrm{AE}$ intensity stabilizes after the elastoplastic transition and allows for the statistical analysis, although a possible effect of overlapping of avalanche-like processes on their statistics should be borne in mind [52]. This approach is supported by recent experimental investigation of AE accompanying plastic deformation of various metals, which shows robustness of the amplitude statistics with regard to the parameters used to 
individualize the events [53]. For these reasons, only amplitude distributions will be considered in the following.

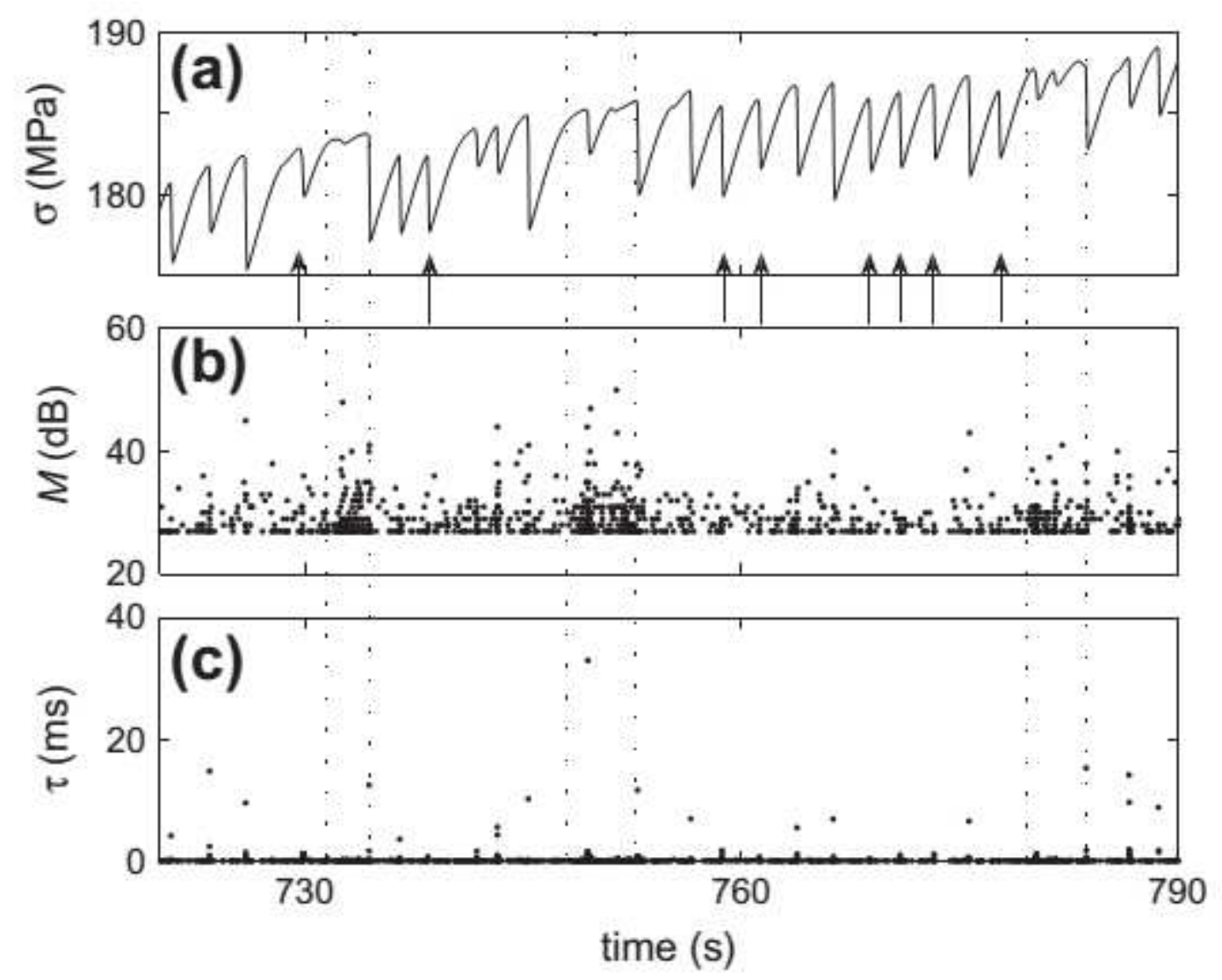

Fig. 11. Same as Fig. 8 for type B serrations at $\dot{\varepsilon}_{a}=2 \times 10^{-4} \mathrm{~s}^{-1}$. Arrows point to stress drops which are not accompanied with $\tau$ bursts. Dotted rectangles show intervals of increased AE activity during transition periods between groups of regular stress serrations.

Two approaches to the calculation of $\mathrm{AE}$ amplitude distributions are reported in the literature. The former is inspired by earthquakes statistics, which obeys a power law in the form of the Gutenberg-Richter relationship relating the number of earthquakes to their magnitude, roughly corresponding to the logarithmic peak amplitude $M$ [54]. In this case, the distribution of the asrecorded amplitudes is directly calculated. Utilizing the logarithmic amplitude means, however, that power-law behavior is assumed a priori in the analysis. In the latter, it was argued [28] that the peak amplitude $A$ of the acoustic event expressed in voltage units or, more specifically, its squared value $A^{2}$, provides a physically based measure of plastic activity reflecting the energy dissipated by the plastic processes in a deforming sample. Both approaches were used in the present work, and led to consistent results. Below, the probability density $P\left(A^{2}\right)$ is presented in order to provide a basis for comparison with the literature data on pure materials. 
The main result of this analysis is that the distributions found for AE obey power laws in all deformation conditions, including the type $\mathrm{B}$ and type $\mathrm{C}$ cases, although the latter are characterized by peaked distributions of stress serrations. Examples of such dependences are presented in Figs. 12 and 13, which show that the power law covers more than two (up to three) orders of magnitude of the variable $A^{2}$. The shortest scaling range, less than 1.5 orders of magnitude, is seen at the strain rate of $6 \times 10^{-3} \mathrm{~s}^{-1}$, for which the statistics is most strongly depleted because of the merging effect. As also follows from these figures, the power-law exponent $\alpha_{A E}$ is virtually unaffected by the recording device settings, in conformity with the suggestion that the information lost because of the events merging does not transform the scale-invariant statistics. Some deviation from the powerlaw dependence can be seen at the largest scales for the coarse time settings. It is most likely due to some overlapping effect on the AE event amplitudes. More details on the investigation of the contribution of the overlapping of the AE events are given in Ref. [46].

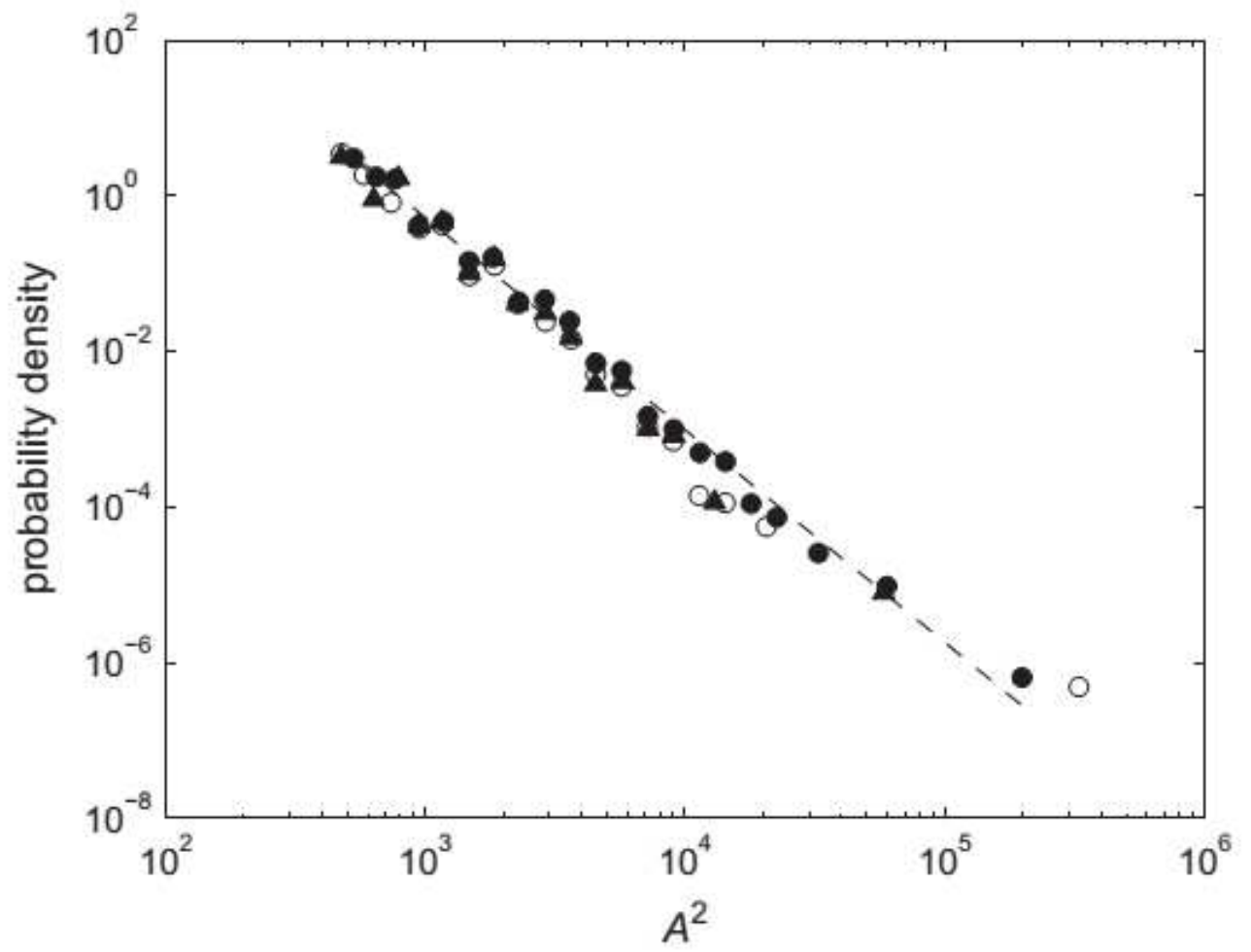

Fig. 12. Probability density function for squared amplitude of $A E$ events collected in the same strain range for three different samples deformed at driving strain rate $\dot{\varepsilon}_{a}=2 \times 10^{-5} \mathrm{~s}^{-1}$. Solid and open circles correspond to the same choice of the device settings, HDT $=$ HLT $=300 \mu \mathrm{s}$; triangles show the data for the set HDT $=$ HLT $=40 \mu \mathrm{s}$.

The robustness of $\alpha_{A E}$ allows examination of its strain and strain-rate dependence. Our first attempt at such an analysis was made in Ref. [24]. The procedure has been further improved since. Its first step consists in searching the time intervals during which both AE and stress fluctuations 
look visually steady. This partition is then refined by varying the width of the intervals and repeating the calculation of the distribution functions until finding intervals where $\alpha_{A E}$ remains approximately constant (cf. Ref. [40]). This representation is summarized in Fig. 14. The intervals of statistically stationary behavior are designated by rectangles, the height of which scales with the least square error of the $\alpha_{A E}$ calculation. It can be recognized that the exponent $\alpha_{A E}$ evolves during the deformation process. Depending on the driving rate, it varies between 1.5 and slightly more than 2 at the onset of plastic deformation. During the unstable flow, $\alpha_{A E}$ takes on higher values, ranging roughly from 2 to more than 3. Relatively similar values of $\alpha_{A E}$ are observed in the type A regime for all samples (Fig. 14a), although the large error caused by overlapping in the high strain-rate tests renders quantitative conclusions difficult. The error of $\alpha_{A E}$ determination is generally lesser at smaller strain rates. Considerable variations of $\alpha_{A E}$ with strain and from sample to sample can be noticed in the type B regime (Fig. 14b). This observation is in agreement with the conclusions on the sensitivity of dislocation dynamics to the microstructure, drawn in Ref. [18] from the multifractal analysis of stress serrations of type B. Finally, $\alpha_{A E}$ decreases in the vicinity of necking.

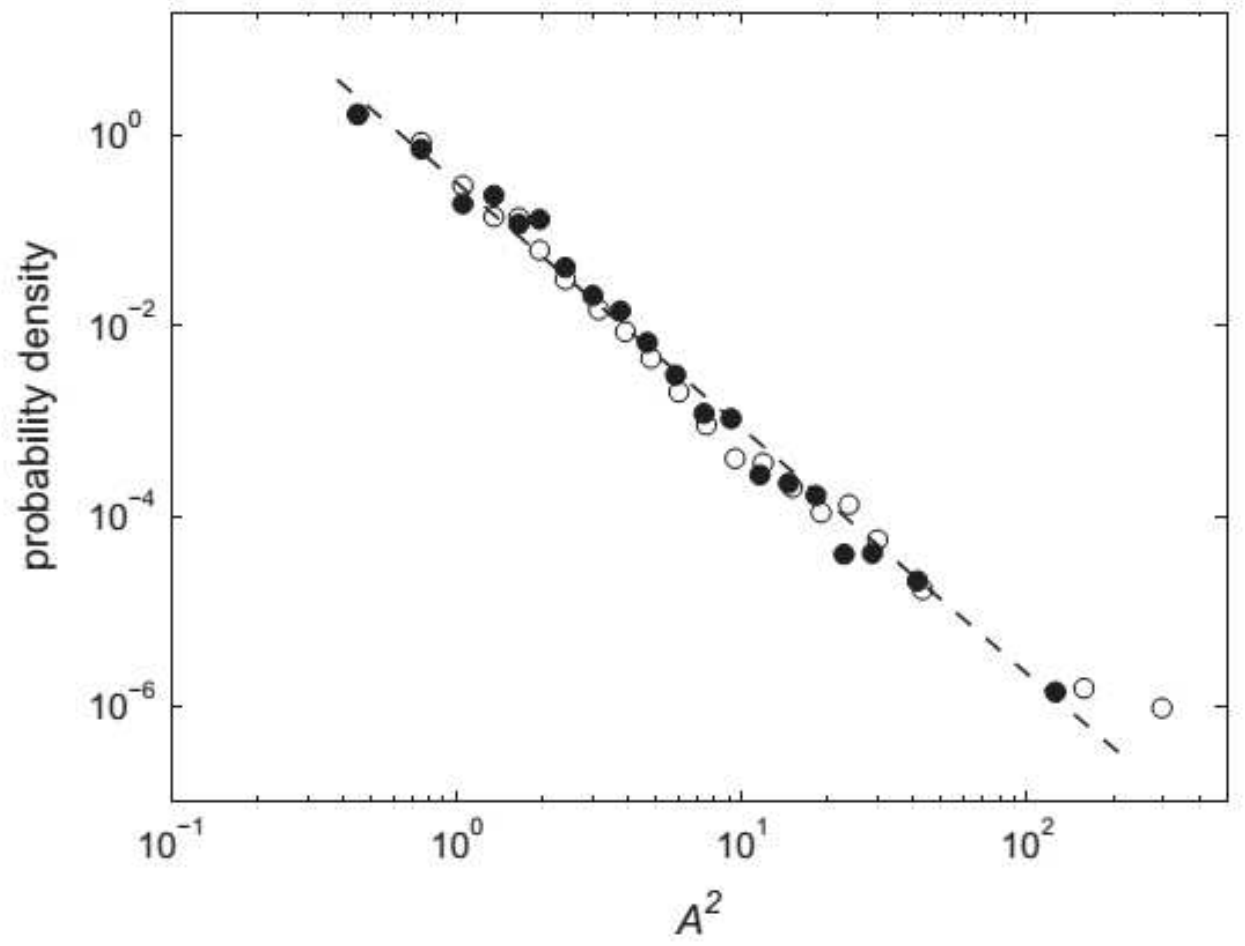

Fig. 13. Probability density function for $A^{2}$ in the case of modification of the device settings during a test at $\dot{\varepsilon}_{a}=6 \times$ $10^{-4} \mathrm{~s}^{-1}$. The data are gathered in the adjacent time intervals (just before and just after the change in the settings). Solid symbols: $\mathrm{HDT}=\mathrm{HLT}=300 \mu \mathrm{s} ;$ open symbols: $\mathrm{HDT}=\mathrm{HLT}=30 \mu \mathrm{s}$.

\section{Discussion and conclusions}




\subsection{Scale-invariant behavior of $\mathrm{AE}$}

The comparative analysis of the deformation curves and the concomitant AE reveals a complex multiscale character of the plastic activity during jerky flow, which is found to depend not only on the experimental conditions, but also on the scale of observation. The macroscopic stress serrations show statistical properties, consistent with the results of previous investigations $[2,10,16,18]$. Peaked and bimodal histograms are observed at low and intermediate driving strain rates. They are associated with essentially static deformation bands, increasingly correlated with increasing $\dot{\varepsilon}_{a}$. Power-law distributions, a hallmark of scale-free, avalanche-like processes, are found in high driving strain-rate tests, when the spatial correlation is so strong that the strain pattern transforms into bands propagating along the sample.

(a)
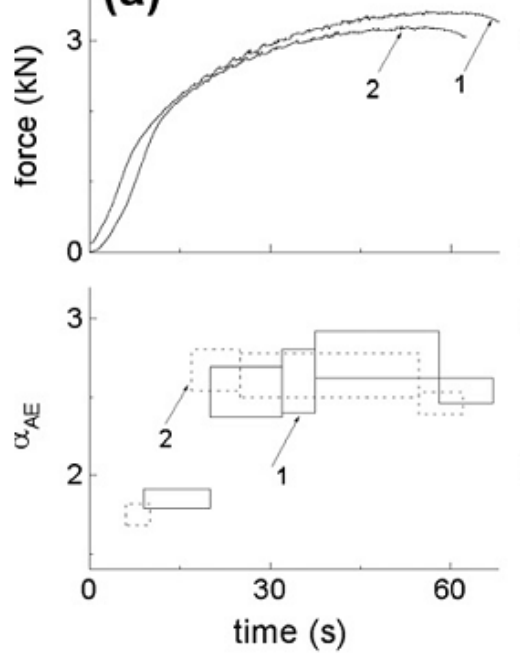

(b)
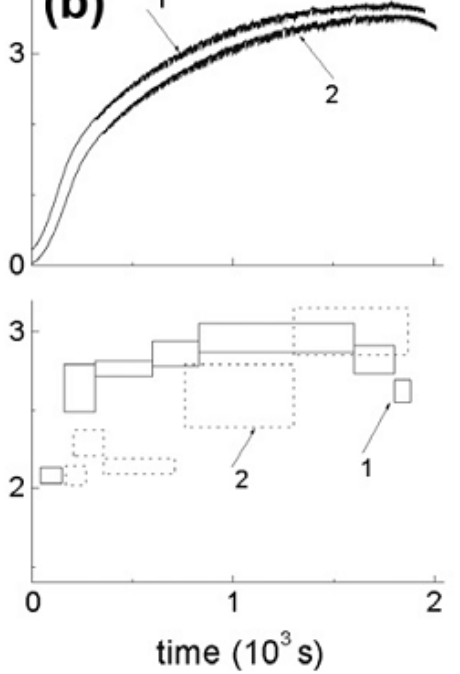
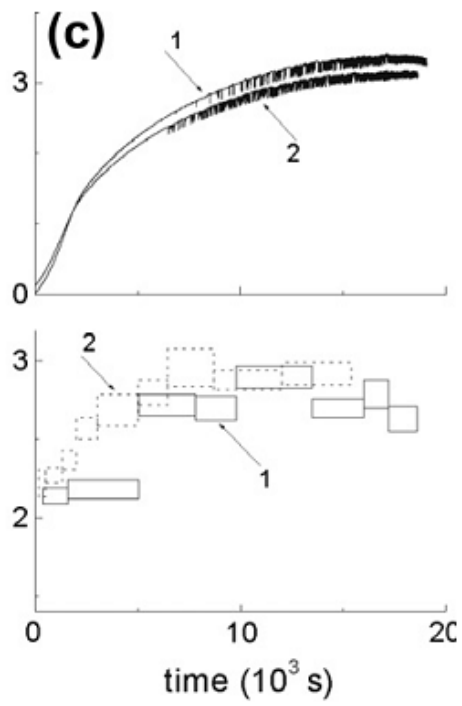

Fig. 14. Force vs. time curves and variation of the power-law index $\alpha_{A E}$ for the AE energy distribution. (a) $\dot{\varepsilon}_{a}=6 \times$ $10^{-3} \mathrm{~s}^{-1}$; (b) $\dot{\varepsilon}_{a}=2 \times 10^{-4} \mathrm{~s}^{-1}$; (c) $\dot{\varepsilon}_{a}=2 \times 10^{-5} \mathrm{~s}^{-1}$. To avoid overcharging the figure, results only for two samples are presented for each value of the driving strain rate; the overlapping deformation curves are deliberately shifted along the ordinate axis..

A qualitatively different behavior is detected at the finer scale uncovered by the AE technique, namely: AE is characterized by power-law statistics in all experimental conditions. This result pertains not only to the various types of PLC behavior (types A, B and C), but also to the plastic activity below the critical strain for PLC instability. The power-law statistics is also found for the low-amplitude stress serrations that are resolved in low strain-rate tests, and correspond to an intermediate scale between the small plasticity events producing AE and macroscopic stress drops. Such persistence of a statistical behavior certainly testifies to the invariant nature of the deformation processes at play during both stable and unstable deformation, and implies an inherently intermittent scale-invariant character of the plastic activity in all cases. This conjecture is 
corroborated by the results of the multifractal analysis in Ref. [40]. On the other hand, it raises the question as to why the stress drops associated with the macroscopic instability show a different statistical behavior in certain experimental conditions. The answer to this question is given by confronting the series of amplitudes and durations of AE events with the stress fluctuations (Figs. 8, 9 and 11), which uncovers a tendency to synchronization of the AE events at the instants of stress drops at low enough $\dot{\varepsilon}_{a}$. It follows that the PLC bands are not uniform objects that could be considered as elementary events, but are rather aggregates of dislocation avalanches. This statement is consistent with recent observations by optical methods of the development of deformation bands, although at a slower time scale [55-58]. It complements at large scales the intermittency and scale invariance found at a very fine scale in the waveforms of individual AE events [37]. The possible mechanism leading to such behavior will be discussed below in Section 4.2.

The ubiquitous character of the power-law statistics of AE calls for a comparison with the literature data for pure materials [23,28,29]. A universal exponent $\alpha_{A E}=1.5-1.6$ was observed in these references for single crystals of various materials with hexagonal and cubic crystal structure. The value of $\alpha^{*}=1.35$ was found for the amplitude distribution of AE in ice polycrystals, which corresponds to $\alpha_{A E}=\left(\alpha^{*}+1\right) / 2 \approx 1.2$ for the energy distribution [33]. The lower value of $\alpha_{A E}$ in ice polycrystals, which implies a larger fraction of big events, was attributed to a dual role of grain boundaries. Namely, although grain boundaries are obstacles to the propagation of slip, large internal stresses generated by dislocation pile-ups promote the activation of dislocation sources in neighboring grains, which may lead to an enhanced probability for large avalanches. A quantitatively different situation is observed in the present work. First, the exponent $\alpha_{A E}$ found for AlMg polycrystals exceeds 1.5 throughout the deformation, suggesting a significantly larger fraction of low-amplitude $\mathrm{AE}$ events. Moreover, $\alpha_{A E}$ increases with strain, except for the latest stage of deformation associated with necking. The discrepancy with the above result [33] can be explained by strong differences in the hardening mechanisms between ice and AlMg polycrystals. Indeed, the virtual absence of forest hardening and the complete absence of solute hardening in ice [59] contrast with the increasing forest dislocation density in AlMg. Forest dislocations contribute to a rather limited size of dislocation avalanches by reducing their free flight distance and leveling off the role of internal stresses. This effect could even be stronger in dynamically strain aging materials owing to the increased strength of obstacles. This conjecture is confirmed by the results presented in Figs. 8 and 9, which illustrate the AE evolution during one test and show that after some strain, the AE events become smaller but form dense merging sequences. The proposed explanation may also account for the decrease in $\alpha_{A E}$ observed in the vicinity of necking, as 
resulting from strain localization. Indeed, the latter enhances simultaneity of slip, which results in the superposition of AE events and a higher probability of large amplitude events.

\subsection{Synchronization of dislocation avalanches}

The occurrence of power-law statistics in the observed behavior is reminiscent of similar features in numerous nonlinear systems of various natures. Well known examples include physical objects, such as the Barkhausen noise in magnetic materials [60] or vortex avalanches in superconductors [61], as well as mechanical or cognate systems, e.g. fracture [42,62], martensitic transformations [63], dry friction [6] or earthquakes [54], and plastic instability due to mechanisms other than the PLC effect $[26,64]$. Several models were proposed in the literature to explain such critical-like behavior, including self-organized criticality, i.e. transition to criticality without finetuning of a control parameter [3], fluctuations near a critical point [65], sweeping of a control parameter of instability [66], etc.

At the same time, the joint occurrence in type $B$ and type $C$ of stress drops with a relatively regular character and of scale-invariant bursts of AE activity prompts us to suggest synchronization of small-scale bursts of plastic activity, a process we see as able to merge the latter into more regular jerks of a macroscopic size. From a generic point of view, synchronization phenomena are observed in large assemblies of coupled oscillators, and may lead to periodic coherent activity of all or part of the oscillators [4]. It is generally accepted that the oscillatory character of each element is a necessary condition for synchronization. In the case of plastic deformation, the latter is warranted by the stick-slip behavior of the thermally activated motion of dislocations through an array of obstacles. It is natural to suggest that the synchronization of plastic activity occurs via elastic waves. Indeed, a burst of plastic activity in some part of the sample results in the emission of strong elastic waves, which transmit the information to other dislocation groups, possibly triggering their activity. In the meantime, the mechanism is resettled in a state where it can be fired again.

Of special interest for the substantiation of the above interpretation are models allowing for a transition between scale-free and synchronized behaviors. The generic models studied in Ref. [67] exhibit avalanches with all possible sizes (SOC), synchronization, and coexistence of SOC and synchronization when the strength of the spatial coupling and the degree of nonlinearity of the driving force are varied. Specifically, these models find themselves in a SOC state when the nonlinearity of the driving force (more to the point, the downward convexity at threshold) is small and the coupling strength is high. Moving away from these conditions leads to a transition to synchronized behavior. These criteria are consistent with the above interpretation, and with the understanding of the crossover mechanisms between the types A, B, C of PLC behavior, proposed 
qualitatively in Refs. [10,16,18] and quantitatively in Ref. [13]. In these papers, the strength of the coupling is controlled by the plastic relaxation of the internal elastic stresses generated by the strain incompatibilities in the vicinity of the bands. However, the following arguments are general and can be extended to other coupling mechanisms, because their role also consists in strain homogenization in the specimen [2]. At large driving strain rates, the reloading time between stress drops is much smaller than the plastic relaxation time. The relaxation of internal stresses is therefore insignificant, and strength of the spatial coupling is high. Further, the $N$-shaped characteristic function $\sigma(\dot{\varepsilon})$, reflecting the nonlinearity of the driving force, is shallowed at high driving rate [2], which also promotes SOC because the dislocation ensembles constantly find themselves near the threshold of instability. As a result, avalanches of any size are allowed and SOC is observed ${ }^{2}$. Thus, although the merging of the dislocation processes is strong because of their high overall activity, the synchronization plays a minor role in these conditions (cf. Ref. [40]). At low strain rates, both factors change. The internal stress field is effectively relaxed during the reloading time between stress drops, which weakens spatial coupling. The $\sigma(\dot{\varepsilon})$ function now shows sharp downward convexity at threshold, and hence the threshold stress is reached nearly simultaneously in various parts of the specimen. As a result, the nucleation of avalanches occurs at any point, which implies absence of correlation with the previous PLC band, and large avalanches of a typical size are likely. As the loading proceeds, this mechanism repeats itself and gives rise to relaxation oscillations. In concluding this section, we note that the present data qualify the PLC effect as a rare candidate for laboratory illustration of the predictions of models for SOC and synchronization.

\section{Concluding remarks}

The above picture provides new insights into the process of PLC band formation. On the sample surface, the bands are seen as markings resulting from dislocation glide along a bunch of parallel slip planes. The present interpretation, based on the merger of AE events during a stress drop, suggests that slip initiation at different sites is not simultaneous, at the time scale of AE, but is instead a sequential process. In particular, it provides a natural explanation to the puzzlingly small number of $\mathrm{AE}$ hits recorded during the hopping propagation of type $\mathrm{B}$ bands, when each band gives rise to one long avalanche, compared with the strong AE activity observed between bands, when

\footnotetext{
${ }^{2}$ In order to avoid confusion, it should be remarked that high driving strain rate conditions do not contradict the "slow driving" assumption of SOC models [3,6]. Indeed, the highest applied strain rate of $6 \times 10^{-3} \mathrm{~s}^{-1}$ is negligible in comparison with the local plastic strain rate of $1-10^{2} \mathrm{~s}^{-1}$ corresponding to the fast time scale associated with the motion of dislocations unpinned from the solute atoms.
} 
isolated avalanches generate many short AE hits but do not produce sharp stress drops (Fig. 11) (see also Ref. [36]).

Observation of a similar statistical behavior of AE in jerky flow and macroscopically smooth plastic flow suggests a general nature of the self-organization processes in dislocation ensembles in various materials. Nevertheless, the solute alloys present a special case due to the occurrence of dynamic strain aging. The insensitivity of the AE intensity to the occurrence of the PLC instability, as well as the presence of small but noticeable stress fluctuations before its onset, suggest that DSA acts throughout the deformation process and applies to plastic events at all scales. This conjecture renews the interrogations on the critical conditions for the occurrence of a macroscopic instability, the nature of the small stress fluctuations, and the relationship between these distinct scales. In particular, it can be suggested that DSA promotes the synchronization of dislocation avalanches by increasing the resistance to dislocation motion, which is likely to be essential for the onset of macroscopic serrations. More specifically, this conjecture particularly concerns the range of low and intermediate driving strain-rate values, which is known to be characterized by peculiar behavior of $\varepsilon_{c r}[38,39]$.

\section{Acknowledgements}

Two of the authors (T.A.L. and N.P.K.) would like to acknowledge support from CNRS, Université Paul VerlaineMetz, and Région Lorraine for several scientific visits to Metz during 2008-10.

\section{References}

[1] Portevin A, Le Chatelier F. CR Acad Sci Paris 1923;176:507.

[2] Kubin LP, Fressengeas C, Ananthakrishna G. In: Nabarro FRN, Duesbery MS, editors. Dislocations in Solids, vol. 11. Amsterdam: Elsevier Science; 2002. p. 101.

[3] Bak P, Tang C, Wiesenfeld V. Phys Rev A 1988;38:364.

[4] Strogatz SH. Physica D 2000;143:1.

[5] Van den Beukel A. Phys Stat Sol (a) 1975;30:197.

[6] Carlson JM, Langer JS, Shaw BE. Rev Mod Phys 1994;66:657.

[7] Andronov AA, Vitt AA, Khaikin SE. Theory of Oscillators. Oxford: Pergamon; 1966.

[8] Penning P. Acta Mater 1972;20:1169. 
[9] Estrin Y, Kubin LP. J Mech Behav Mater 1989;2:255.

[10] Lebyodkin MA, Bréchet Y, Estrin Y, Kubin LP. Phys Rev Lett 1995;74:4758.

[11] Hähner P, Ziegenbein A, Rizzi E, Neuhäuser H. Phys Rev B 2002;65:134109.

[12] Ananthakrishna G, Valsakumar MC. J Phys D 1982;15:L171.

[13] Kok S, Bharathi MS, Beaudoin AJ, Fressengeas C, Ananthakrishna G, Kubin LP, et al. Acta Mater 2003;51:3651.

[14] Ananthakrishna G. Phys Rep 2007;440:113.

[15] Varadhan S, Beaudoin AJ, Fressengeas C. J Mech Phys Solids 2009;57:1733.

[16] Lebyodkin M, Dunin-Barkowskii L, Bréchet Y, Estrin Y, Kubin LP. Acta Mater 2000;48:2529.

[17] Ananthakrishna G, Noronha SJ, Fressengeas C, Kubin LP. Phys Rev E 1999;60:5455.

[18] Bharathi MS, Lebyodkin M, Ananthakrishna G, Fressengeas C, Kubin LP. Acta Mater 2002;50:2813.

[19] Kugiumtzis D, Kehagias A, Aifantis EC, Neuhäuser H. Phys Rev E 2004;70:036110.

[20] Darowicki K, Orlikowski J, Zielinski A, Jurczak W. Comput Mater Sci 2007;39:880.

[21] Sarkar A, Webber Jr CL, Barat P, Mukherjee P. Phys Lett A 2008;372:1101.

[22] Chmelik F, Ziegenbein A, Neuhäuser H, Lukac P. Mater Sci Eng A 2002;324:200.

[23] Weiss J, Grasso J-R. J Phys Chem B 1997;101:6113.

[24] Bougherira Y, Entemeyer D, Fressengeas C, Kobelev NP, Lebedkina TA, Lebyodkin MA. J Phys Conf Series 2010;240:012009.

[25] Bobrov VS, Lebedkin MA. Fizika Tverdogo Tela 1989;31:120; Bobrov VS, Lebedkin MA. Sov Phys Solid State 1989;31:982.

[26] Bobrov VS, Zaitsev SI, Lebedkin MA. Fizika Tverdogo Tela 1990;32:3060; Bobrov VS, Zaitsev SI, Lebedkin MA. Sov Phys Solid State 1990;32:1176.

[27] Schmitter ED. Phys Lett A 2007;368:320.

[28] Weiss J, Grasso JR, Miguel MC, Vespignani A, Zapperi S. Mater Sci Eng A 2001;309:360. 
[29] Richeton T, Weiss J, Louchet F, Dobron P, Chmelık F. Kovove Mater 2007;45:149.

[30] Weiss J, Richeton T, Louchet F, Chmelik F, Dobron P, Entemeyer D, et al. Phys Rev B 2007;76:224110.

[31] Dimiduk DM, Woodward C, LeSar R, Uchic MD. Science 2006;312:1188.

[32] Zaiser M. Adv Phys 2006;55:185.

[33] Richeton T, Weiss J, Louchet F. Nat Mater 2005;4:465.

[34] Lebyodkin MA, Estrin Y. Acta Mater 2005;53:3403.

[35] Chmelık F, Klose FB, Dierke H, Sachl J, Neuhäuser H, Lukac P. Mater Sci Eng A 2007;462:53.

[36] Reed JM, Walter ME. Mater Sci Eng A 2003;359:1.

[37] Lebyodkin MA, Lebedkina TA, Chmelik F, Lamark TT, Estrin Y, Fressengeas C, et al. Phys Rev B 2009;79:174114.

[38] Balik J. Mater Sci Eng A 2001;316:102.

[39] Lebedkina TA, Lebyodkin MA. Acta Mater 2008;56:5567.

[40] Lebyodkin MA, Kobelev NP, Bougherira Y, Entemeyer D, Fressengeas C, Lebedkina TA, et al. Acta Mater 2012;60:844.

[41] Kral R, Dobron P, Chmelik F, Koula V, Rydlo M, Janecek M. Kovove Mater 2007;45:159.

[42] Aue J, De Hosson JThM. J Mater Sci 1998;33:5455.

[43] Fressengeas C, Beaudoin AJ, Entemeyer D, Lebedkina T, Lebyodkin M, Taupin V. Phys Rev B 2009;79:014108.

[44] Lebyodkin MA, Lebedkina TA. Phys Rev E 2008;77:026111.

[45] Chatterjee A, Sarkar A, Barat P, Mukherjee P, Gayathri N. Mater Sci Eng A 2009;508:156.

[46] Bougherira Y. PhD report, Université Paul Verlaine-Metz; 2011.

[47] Kerteész J, Kiss LB. J Phys A 1990;23:L433.

[48] Thevenet D, Mliha-Touati M, Zeghloul A. Mater Sci Eng A 1999;266:175.

[49] Abbadi M, Hähner P, Zeghloul A. Mater Sci Eng A 2002;337:194. 
[50] Kumar J, Ananthakrishna G. Phys Rev Lett 2011;106:106001.

[51] Vinogradov A. Scripta Mater <http://dx.doi.org/10.1016/j.scriptamat.2012.01.053>.

[52] White RA, Dahmen KA. Phys Rev Lett 2003;91:085702.

[53] Shashkov IV, Lebyodkin MA, Lebedkina TA, Dobron P, Chmelik F, Kral R, Parfenenko K, Mathis K. Acta Phys Pol 2012, in press.

[54] Gutenberg B, Richter CF. Bull Seismol Soc Am 1942;32:163.

[55] Shabadi R, Kumara S, Roven HJ, Dwarakadasa ES. Mater Sci Eng A 2004;364:140.

[56] Jiang Z, Zhang Q, Jiang H, Chen Z, Wu X. Mater Sci Eng A 2005;403:154.

[57] Shibkov AA, Zolotov AE. JETP Lett 2009;90:370.

[58] Casarotto L, Dierke H, Tutsch R, Neuhäuser H. Mater Sci Eng A 2009;527:132.

[59] Shearwood C, Whitworth RW. Phil Mag 1991;64:289.

[60] Cote PJ, Meisel LV. Phys Rev Lett 1991;67:1334.

[61] Matizen EV, Ishikaev SM, Oboznov VA. J Exp Theor Phys 2004;99:1065.

[62] Canneli G, Cantelli R, Cordero F. Phys Rev Lett 1993;70:3923.

[63] Vives E, OrtinJ,Manosa L, Ràfols I, Pérez-Magrané R, Planes A. Phys Rev Lett $1994 ; 72: 1694$.

[64] Lebyodkin MA, Dunin-Barkovskii LR, Lebedkina TA. JETP Lett 2002;76:612.

[65] Sethna JP, Dahmen K, Kartha S, Krumhansl JA, Roberts BW, Shore JD. Phys Rev Lett $1993 ; 70: 3347$.

[66] Sornette D. Phys Rev Lett 1994;72:2306.

[67] Pérez CJ, Corral A, Diaz-Guilera A, Christensen K, Arenas A. Int J Mod Phys B 1996;10:1111. 